\title{
Integrating isotopes and documentary evidence: dietary patterns in a late medieval and early modern mining community, Sweden
}

\author{
Ylva Bäckström $^{1}$ • Jan Mispelaere ${ }^{2}$ - Anne Ingvarsson ${ }^{3}$ - Markus Fjellström ${ }^{4}$. \\ Kate Britton ${ }^{5,6}$
}

Received: 10 February 2017 / Accepted: 30 May 2017 /Published online: 12 July 2017

(C) The Author(s) 2017. This article is an open access publication

\begin{abstract}
This study explores the relationship between dietary patterns and social structure in a pre-industrial mining community in Salberget, Sweden c. 1470 to 1600 A.D. using a combination of different research approaches and tools, including archaeology, osteology, bone chemistry and history. The correlation between demographic criteria (sex and age) and archaeological variables (burial type and burial location) shows that Salberget was a highly stratified community.
\end{abstract}

Ylva Bäckström

ylva.backstrom@ark.lu.se

Jan Mispelaere

Jan.mispelaere@riksarkivet.se

Anne Ingvarsson

Anne.ingvarsson@gustavianum.uu.se

Markus Fjellström

Markus.fjellstrom@arklab.su.se

Kate Britton

k.britton@abdn.ac.uk

1 Department of Archaeology and Ancient History, LUX, Lund University, Box 192, 22100 Lund, Sweden

2 The Swedish National Archives, Box 12541, 102 29 Stockholm, Sweden

3 Gustavianum, Uppsala University Museum, Akademigatan 3, 753 10 Uppsala, Sweden

4 Archaeological Research Laboratory, Department of Archaeology and Classical Studies, Stockholm University, 106 91 Stockholm, Sweden

5 Department of Archaeology, University of Aberdeen, St. Mary's Building, Elphinstone Road, Aberdeen, Scotland AB24 3UF, UK

6 Department of Human Evolution, Max Planck Institute for Evolutionary Anthropology, Deutscher Platz 6,

04103 Leipzig, Germany
Group diets were investigated through analyses of stable isotopes (carbon, $\delta^{13} \mathrm{C}$, and nitrogen, $\delta^{15} \mathrm{~N}$ ) of bone collagen from a sub-sample of individuals buried at the site $(n=67)$, interpreted alongside data from human dental lesions and deficiencies, animal bone waste and information on eating habits extracted from the extensive historical documents regarding mining activities at Salberget. These integrated analyses provide a clear association between social status and diet and confirm that social status, and to a lesser extent sex, gender and age, likely governed food choice and opportunity in this diverse community.

Keywords Late medieval/early modern · Preindustrial · Identities · Diet · Documentary sources · Bioarchaeology · Isotopes $\cdot$ Zooarchaeology $\cdot$ Dental palaeopathology

\section{Introduction}

In modern society, there is a close link between diet and social identity, for example, as reflected in dietary differences with economic status, religion, education, gender and age (e.g. Joyce 2005; Knudson and Stojanowski 2008; Zvelebil and Weber 2013). Such socio-cultural and socio-economic differences existed in late medieval and early modern society and were also likely to have influenced individual and group dietary habits (see for example Woolgar et al. 2006). While these aspects of past lives may be difficult to discern using traditional archaeological approaches, bioarchaeological — and specifically stable isotopic - approaches, in combination with documentary sources, have the potential to reveal insights into past dietary habits on individual- and population-scale levels.

A large number of studies have demonstrated the ways which stable isotope data can illuminate medieval dietary habits in European populations, and social expressions of diet, 
including studies from Britain (e.g. Müldner 2005; Bell et al. 2009; Müldner et al. 2009; Lamb et al. 2012), Scandinavia (e.g. Kjellström et al. 2009; Yoder 2010), the Iberian Peninsula (e.g. Salazar-Garcia et al. 2014; Alexander et al. 2015; Jiménez-Brobeil et al. 2016) and Southern (e.g. Reitsema and Vercellotti 2012; Iacumin et al. 2014; Ciaffi et al. 2015) and Central Europe (e.g. Reitsema et al. 2010; Olsen et al. 2016). In recent years, there has also been a growing body of isotopic research undertaken on early modern materials from these regions (e.g. Johansen et al. 1986; Mays 1997; Richards et al. 2002; Müldner 2005; Müldner and Richards 2007a, b; Salesse et al. 2013; Quintelier et al. 2014; Jørkov and Gröcke 2016), although these are more limited in number, mainly due to the lack of excavations or skeletal assemblages not being routinely retained for long-term study from such sites. In Sweden, for example, there are few early modern studies to date (for exceptions, see Lidén and Nelson 1994; Fjellström 2011; Arcini et al. 2014), and until now, these have not included pre-industrial communities. However, this period is fertile ground for the development of further research, not least due to a vast archival material and an extensive historical literature discussing diets during this period, with major works on diet in early modern and industrializing Sweden being those of Morell (1986, 1989, 2011) and Utterström (1978).

The aim of this paper is to examine if sociocultural and/or socioeconomic differences, evidenced by burial customs, burial locations and demography, can be correlate with diet in a late medieval/early modern, pre-industrial, pre-industrial skeletal population from Sweden - the Salberget mining community (Fig. 1). The animal bone waste from the archaeological excavations and the vast contemporary archival material from Salberget give a rare opportunity for an in-depth interpretation of diet through dietary isotopes and the integration of isotope data with other evidence types. Specifically, we examine whether or not the dietary habits identified through both stable isotope analyses and literary sources correlate with implied differences in social and other group identities recognised in the Salberget cemetery from burial customs, burial locations and demography, along with other aspects such as sex or age group.

\section{Reconstructing late medieval and early modern diets in Sweden: stable isotope and documentary approaches}

The stable isotope analysis of human (and animal) bone collagen as a means of reconstructing diet is now relatively common in archaeological studies since the first applications in the late 1970s and early 1980s (see review in Makarewicz and Sealy 2015). The stable isotope composition of bone collagen reflects the isotopic composition of protein consumed during life (DeNiro and Epstein 1978, 1981; Schoeninger and DeNiro 1984), albeit with minor contributions from other dietary macronutrients (Warinner and Tuross 2009; Froehle et al. 2010). As bone collagen turnover rates can vary depending on the osteological element, the stable isotope ratio of human bone collagen reflects dietary protein intake consumed over the last 10-30 years (Ambrose and Norr 1993; Wild et al. 2000; Hedges et al. 2007).

In temperate and more northerly regions, stable carbon isotope value $\left(\delta^{13} \mathrm{C}\right)$ measurements of human bone collagen are most commonly used to discriminate between marine and terrestrial dietary protein sources (Schoeninger and DeNiro 1984), and nitrogen isotope ratios $\left(\delta^{15} \mathrm{~N}\right)$ can be useful in determining the trophic level of protein consumed. The stable nitrogen is measured according to the standard ambient inhalable reservoir (AIR), and its $\delta^{15} \mathrm{~N}$ values increase in general by $3-5 \%$ with each step up the food chain (e.g. DeNiro and Epstein 1981; Schoeninger et al. 1983; Ambrose 2000; Bocherens and Drucker 2003). As marine and freshwater food chains tend to be longer than terrestrial ones, they tend to have higher end values (Schoeninger and DeNiro 1984). While $\delta^{13} \mathrm{C}$ measurements of human bone collagen can also be utilised to explore the prevalence of $\mathrm{C} 4$ (tropical) grasses in the diet (such as maize or even animals foddered on maize or other $\mathrm{C} 4$ plants, see review in Makarewicz and Sealy 2015), these are unlikely to have played a major dietary role in Scandinavia in this period. However, $\delta^{13} \mathrm{C}$ measurements of human bone collagen can illuminate the relative proportion of marine foods in the diet (Schoeninger and DeNiro 1984: 625), and it has been estimated that stable carbon isotope ratios for human bone collagen for diets based around the Baltic Sea region could be anticipated to range from -21 to $-14 \%$, where the least negative value is the most marine, and the lowest being dominated by terrestrial protein sources (Lidén and Nelson 1994: 18). Therefore, the measurement of $\delta^{13} \mathrm{C}$ and $\delta^{15} \mathrm{~N}$ values of archaeological human and animal samples can allow the reconstruction of trophic relationships, the identification of marine resources in the diet, and the determination of likely sources of human dietary protein.

While stable isotope analysis of human bone collagen is a powerful tool for providing both individual dietary habits and population level trends, bone collagen stable isotope ratios predominately reflect only the dietary protein consumed. Therefore, alternative sources of biomolecular, anthropologi$\mathrm{cal}$, archaeological or historical evidence are often required to provide a fuller or more nuanced picture of past diet. When used in combination with 'bulk' bone stable isotope approaches, these can provide complementary forms of data and a more complete picture of past dietary habits. The study of animal bones, for example, provides evidence of not only what species were being targeted but also the ways in which they may have been butchered and otherwise prepared and, above all, what the culling patterns can tell about animal husbandry, production and consumption. However, disposal 
Fig. 1 Map of Northern Europe and Sweden with Sala/Salberget, Västerås and the Bergslagen area (grey). Sala is located c. $120 \mathrm{~km}$ west of the Baltic Sea and c. $40 \mathrm{~km}$ north of the large freshwater lake of Mälaren

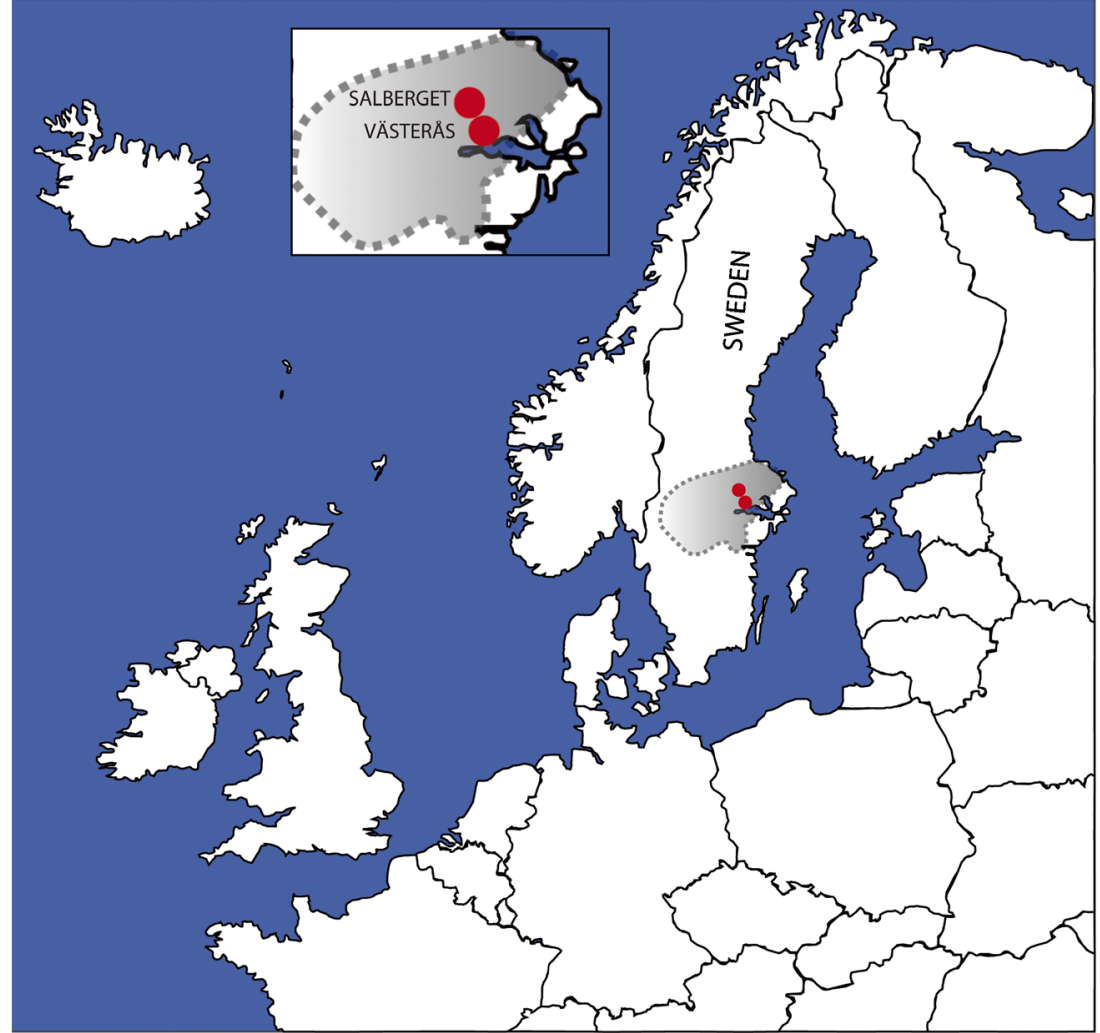

practices in the past, along with modern excavation techniques and post-excavation analyses, can influence the quality and quantity of datasets available. Skeletal pathologies, particularly dental pathologies, can also provide useful complementary evidence of diet and health when combined with isotope data. For example, dental caries might be correlated with diets high in carbohydrates, although - as caries are caused by multiple factors and also correlate with age-interpretations are rarely straightforward (see for example Lukacs 2011). Calculus thrives in an alkaline environment and has therefore been linked to protein-rich diets (Hillson 1979: 150), although this relationship has been questioned (see for example, Whittaker et al. 1998; Lieverse 1999). Moreover, dental pathologies such as caries and calculus are greatly influenced by cultural habits affecting oral hygiene practices in a population and also by the infrastructure of the society, which determines access to dental care (see for example Novak et al. 2012). Socio-cultural differences in a society could also be studied through childhood stress (including changes in dietary habits and malnutrition) evidenced by linear enamel hypoplasia (disturbances of enamel formation) (see for example Palubeckaité et al. 2002; Lewis 2013). Documentary (historical) evidence is another valuable source of information about diet but is highly variable, depending on the underlying purpose of the documents (e.g. for taxation purposes, as payment rolls, or as a display of wealthy citizens feasting habits etc.), and also on the entities and social categories of people mentioned in the texts. In general, there is a bias in the written documents emphasizing those in power, i.e. the landowning classes.

In general terms, different classes in society in late and post-medieval Europe are thought to have had different dietary habits, although sub-identities (based on age, gender or maybe special groups like ecclesiastical communities or nascent industrial settlements, such as those around mines) also governed food choices within the social groupings and resulted in a much more heterogeneous diet on a detailed level (see for example Woolgar 2001, 2006). However, the diet for most people in medieval Sweden was based on unleavened bread, porridge and gruel, accompanied by salt fish, stockfish and some meat, with beer and ale widely consumed. The diet of the upper classes was generally more varied (Adamson 2004: 48-49; Woolgar 2006: 196; Morell 2011). Cultural ideas of what to eat created food hierarchies - where meat was almost always prized and plants less so - and also dictated which foodstuffs should be consumed, and which avoided (and by whom), although compliance with the written or unwritten rules varied between and within groups of society. For example, the poor in society could not always afford to obey the regulations (Adamson 2004: 181-182). Religion and related philosophies, as with most aspects of medieval and early modern life (Wåghäll Nivre and Larsson 2001: 122), would have had a profound influence on diet, both with regards to the body itself (i.e. the humoral theory) and society more broadly (Adamson 2004: 205, 233; Woolgar 2006: 191, 198). While 
the seasonal availability of foodstuffs would have limited some choices, the menu was also rigorously governed by the Christian calendar and divided into meat, lean (or fish) and feast days (Adamson 2004: 188, 233). The Catholic Church prescribed abstinence from the meat of warm-blooded animals, eggs and dairy products during approximately a third of the year. Meat was often substituted by fish, and sometimes the menu consisted of bread, legumes and vegetables. The number of fasting days varied throughout the medieval period, but Fridays and Saturdays throughout the year seem to have been lean days. The longest fasting period, Lent, occurred before Easter and lasted for 40 days (for more detail, see Adamson 2004: 188-189). During the sixteenth century, the Reformation reduced the number of fasting days (see for example Malmstedt 1994), although it was unlikely that this change was instantaneous and probable that it took some time for people to adjust to this new dietary regime.

\section{Materials}

\section{The Salberget mining community}

Salberget silver mine is situated in the mining district of Bergslagen, central Sweden, where preindustrial mining started in several locations during the high and late middle ages (see for example Meurman 2000) (Fig. 1). The mine attracted workers from across the country and abroad, and a large village (Salberget) evolved nearby (Fig. 2). In the midsixteenth century, the mining village was divided into four blocks with 294 taxpaying households of at least two people and 33 singles. At the same time, the nearest town to the south, Västerås, had around 950 inhabitants (Jansson 1963: 40-45; Institute of Urban History 2008).

Väsby royal estate served as the local hub of the silver mining district, the headquarters for mining operations and as the administrative centre for the Crown, collecting taxes from the entire region (Sala county) (Fig. 2). The estate imported goods, produced food and to some extent acted as a supplier of basic goods to their employees. In addition, a large part of their wages were paid in-kind (in natura) (Mispelaere and Lindström 2015), and some were paid through everyday meals served at Väsby estate. For instance, in AD 1570, approximately 200 people received a meal through Väsby estate (Account books for Sala and Väsby demesne 1570: 3A).

While the town privileges for Sala were not ratified until AD 1624, the Crown had already provisioned the mining community with particular trading rights and a free market in the sixteenth century. Free-trade markets were held in the mining village two days a week from AD 1535 onwards. In general, the sale of goods in rural areas was extremely limited. Exceptions were made in Salberget, where trade by non- merchants, especially the sale of victuals by smallholders, famers and peddlers, was permitted and stimulated to favour the local economy and provisioning of supplies in the mining areas (Privileges of Salberget 2/7 1530 and 18/7 1535). The most common food sold at the village market was fish (rarely fresh) and hops (Jansson 1963: 7-36). A large amount of cereal was transported from the southern plains up to the mining area and the Väsby estate, along with salt and marine fish from Norway, Finland and from Gävle, on the Swedish east coast (Printed Royal decree from 1526; Account books for Sala and Väsby demesne, 1544-45, 1569-71, 1594). Extensive regular food deliveries were necessary, not only to dissuade workers from moving away from the village due to poor living conditions/limited resources but also to ensure that the workers were healthy, fit to work and ultimately increase the Crown's profits from mining. Besides the peddlers, the local traders and merchants, the Väsby estate provided the population in the village with foodstuff by selling supplies. A large part of the population could, in this way, buy necessities and basic commodities even during tough years with bad harvests. ${ }^{1}$ Studies on eighteenth century iron works have similarly pointed out that, in comparison to the farms in the countryside, these kinds of communities were buffered against bad times due to the Crown's direct involvement and interest in keeping the work going (see discussion in Sundin and Tedebrand 2008).

\section{Documentary sources}

There are exceptionally well-preserved and detailed archives from the sixteenth century onwards, regarding administration, finances and, to a lesser extent, the social conditions at the Salberget silver mine. Sources from both tax authorities, Väsby royal estate and the mine are preserved, such as accounts, reports, correspondence, livestock and tax records, enabling us to some extent to follow the influx of traded foodstuffs in the region from the AD 1530s onwards and the local production of foodstuffs at Väsby estate, by the villagers and by the parishioners.

Tax registers and account books from the AD 1500s and early 1600 s give a picture of the goods delivered, declared and sold at markets in the mining village as well as the food cooked and consumed at Väsby estate. The records also illustrate what kind of goods and foodstuffs were not grown and processed locally. Above all, the documents (in particular food distribution records and the employees' personal account books) provide detailed information about the meals served (as part of the salary), the employees at Väsby demesne and the amount of food distributed to different social groups, for

\footnotetext{
${ }_{1}$ For instance $\mathrm{AD}$ 1602-03, a period with repeated bad harvests mentioned in: Account books and payrolls 1602-03; Salbergets accounts. 1602-03; see also Mispelaere and Lindström 2015.
} 
Fig. 2 Salberget village and cemetery, the mining area, Väsby royal estate, the two contemporary parish churches, Sala town (founded in AD 1624 and succeeding the mining village) and rivers and lakes in the surrounding region

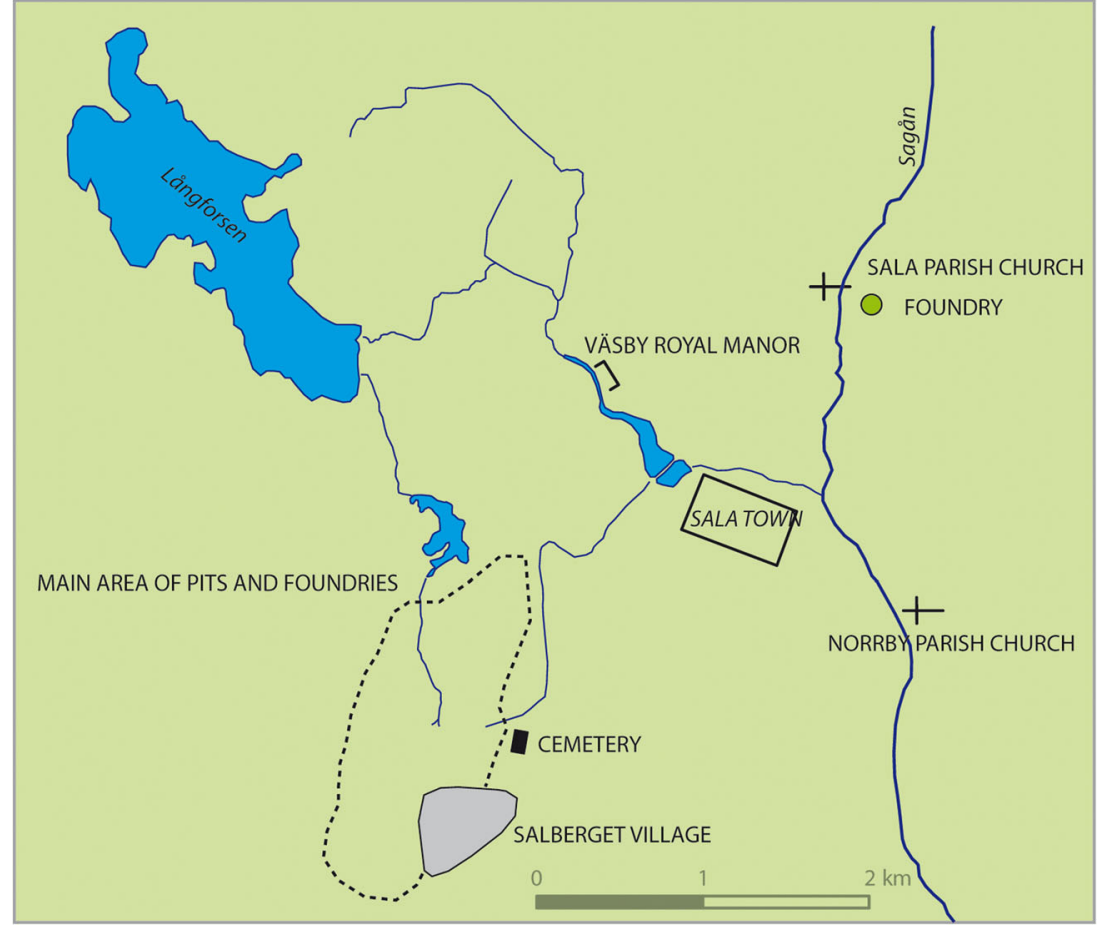

instance the different groups of workers at the mine, charcoalburners, prisoners, craftsmen, clerks and servants at the estate. The accounts and calculations for AD 1570-1571, which we have used as one of our main sources, are extremely detailed, for example.

\section{The cemetery excavations}

In 2004, a small excavation in the mining cemetery revealed the foundation of a church/chapel to the north and 103 patches of darker soil indicating burials (Onsten-Molander and Jonsson 2005). The borders of the cemetery to the north, south and possibly west were established (Fig. 3). No burials were found north of the church or under the church floor. Two years later, a project started with the aim to investigate how the living conditions in a mining community affected the health of the population. Excavations of the cemetery took place in 2008, 2009 and 2011 (Bäckström et al. 2009; Bäckström and Ingvarsson-Sundström 2010, 2012). Around a fifth of the cemetery was excavated and 102 skeletons analysed osteologically by Anne Ingvarsson-Sundström and Ylva Bäckström (Table 1). Standard methods were applied in skeletal sex and age assessments (Buikstra and Ubelaker 1994; see also Bäckström and Price 2016).

The graves were consistently laid out in a Christian manner (head to the west, feet to the east), but burial customs across the cemetery varied. A group of men (predominantly younger men) was buried without coffins in the southern part of the churchyard (trenches B2 and C1). Other men, along with women and children of different ages, were buried in wooden coffins in other areas of the cemetery (Fig. 3). The earthen burials mainly contained multiple bodies interred in everyday clothes versus single burials in coffins with the bodies wrapped in shrouds. One of the earthen buried males was buried with an iron collar still attached to his neck, an item used as punishment for various offenses (see Bäckström and Price 2016: 156, for more detail). A large number of earthen buried males and juveniles showed evidence of unhealed fractures (Price et al. 2017). The manner of burial, clothing and grave goods (including the iron collar) and prevalence of fractures suggest this group was set apart from others buried in the cemetery and may have been of lower socio-economic status. In light of the iron collar in particular, it seems likely these individuals could be the prisoners who were brought to work in the mine in the sixteenth century, as documented in contemporary historical texts (Boethius 1951: 160; Norberg 1978: 56; Eriksson and Berg 2003).

Coins in three of the earthen burials give terminus post quem date between 1535 and 1568. Stray finds of coins also point mainly to the sixteenth century, which coincides with the chronology of the mining village. No coins were found in the coffin graves, but the few finds retrieved place the burials in approximately the same time interval. Therefore, we think that no major chronological difference existed between the two types of burials and they represent broadly contemporary groups of individuals. 
Fig. 3 Salberget cemetery, excavated trenches $(A-D)$

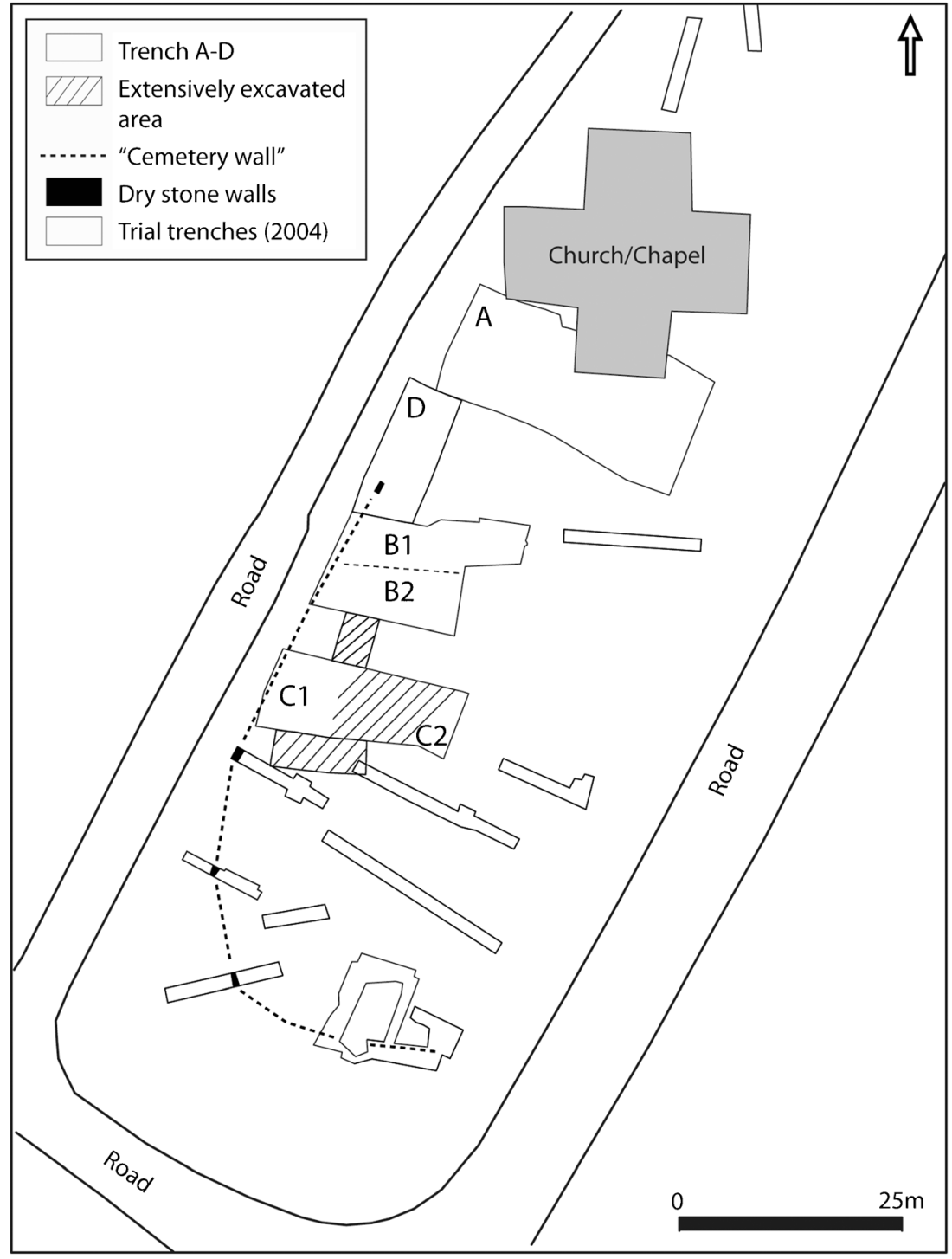

Table 1 Distribution of age and sex, Salberget cemetery $(n=102)$

\begin{tabular}{|c|c|c|c|c|c|c|c|c|c|c|}
\hline & \multicolumn{3}{|c|}{ Coffins, trench A } & \multicolumn{3}{|c|}{ Coffins, trenches B1-2, C2, D } & \multicolumn{4}{|c|}{ Earthen graves, trenches $\mathrm{B} 2, \mathrm{C} 1$} \\
\hline & Females & Males & Indet. & Females & Males & Indet. & Females & Males & Indet. & Total \\
\hline \multicolumn{11}{|l|}{ Children } \\
\hline Younger child $\leq 3$ years & 0 & 0 & 10 & 0 & 0 & 10 & 0 & 0 & 0 & 20 \\
\hline Older child $>3-15$ years & 0 & 0 & 12 & 0 & 0 & 11 & 0 & 0 & 0 & 23 \\
\hline Juveniles $\mathbf{1 5 - 2 0}$ years & 0 & 0 & 3 & 0 & 0 & 2 & 0 & 0 & 10 & 15 \\
\hline \multicolumn{11}{|l|}{ Adults } \\
\hline Young adult 20-35 years & 2 & 0 & 0 & 0 & 3 & 1 & 0 & 5 & 0 & 11 \\
\hline Middle adult $35-50$ years & 3 & 2 & 0 & 1 & 5 & 1 & 0 & 7 & 0 & 19 \\
\hline Old adult $>50$ years & 2 & 4 & 1 & 5 & 1 & 1 & 0 & 0 & 0 & 14 \\
\hline Total & 7 & 6 & 26 & 6 & 9 & 26 & $\mathbf{0}$ & 12 & 10 & 102 \\
\hline
\end{tabular}

Adult age groups according to Buikstra and Ubelaker (1994). For a more detailed description of methods used in determinations of sex and age, see Bäckström and Price (2016) 
Table 2 Prevalence of dental lesions (per individual and per tooth)

\begin{tabular}{|c|c|c|c|c|c|c|c|c|c|c|c|c|c|c|c|c|c|c|}
\hline \multirow[t]{2}{*}{ Cluster A-coffins } & \multicolumn{6}{|c|}{ Caries } & \multicolumn{6}{|c|}{ Calculus } & \multicolumn{6}{|c|}{ LEH } \\
\hline & $\mathrm{Ni}$ & $n$ & $\%$ & $\mathrm{Nt}$ & $n$ & $\%$ & $\mathrm{Ni}$ & $n$ & $\%$ & $\mathrm{Nt}$ & $n$ & $\%$ & $\mathrm{Ni}$ & $n$ & $\%$ & $\mathrm{Nt}$ & $n$ & $\%$ \\
\hline$\leq 3$ years & 4 & 1 & 25.0 & 62 & 1 & 1.6 & 4 & 0 & 0.0 & 62 & 0 & 0.0 & 4 & 0 & 0.0 & 62 & 0 & 0.0 \\
\hline$>3-15$ years & 4 & 2 & 50.0 & 89 & 7 & 7.9 & 4 & 2 & 50.0 & 89 & 9 & 10.1 & 4 & 2 & 50.0 & 89 & 9 & 10.1 \\
\hline Juveniles & 1 & 0 & 0.0 & 32 & 0 & 0.0 & 1 & 1 & 100.0 & 32 & 1 & 3.1 & 1 & 1 & 100.0 & 32 & 22 & 68.8 \\
\hline Young adults & 1 & 0 & 0.0 & 27 & 0 & 0.0 & 1 & 1 & 100.0 & 27 & 1 & 3.7 & 1 & 1 & 100.0 & 27 & 16 & 59.3 \\
\hline Middle adults & 2 & 2 & 100.0 & 30 & 8 & 26.7 & 2 & 1 & 50.0 & 30 & 1 & 3.3 & 2 & 0 & 0.0 & 30 & 0 & 0.0 \\
\hline Old adults & 4 & 3 & 75.0 & 89 & 7 & 7.9 & 4 & 4 & 100.0 & 89 & 28 & 31.5 & 4 & 1 & 25.0 & 89 & 4 & 4.5 \\
\hline Total & 16 & 8 & 50.0 & 329 & 23 & 7.0 & 16 & 9 & 56.3 & 329 & 40 & 12.2 & 16 & 5 & 31.3 & 329 & 51 & 15.5 \\
\hline \multirow[t]{2}{*}{ Cluster B-coffins } & \multicolumn{6}{|c|}{ Caries } & \multicolumn{6}{|c|}{ Calculus } & \multicolumn{6}{|c|}{ LEH } \\
\hline & $\mathrm{Ni}$ & $n$ & $\%$ & $\mathrm{Nt}$ & $n$ & $\%$ & $\mathrm{Ni}$ & $n$ & $\%$ & $\mathrm{Nt}$ & $n$ & $\%$ & $\mathrm{Ni}$ & $n$ & $\%$ & $\mathrm{Nt}$ & $n$ & $\%$ \\
\hline$\leq 3$ years & 5 & 0 & 0.0 & 62 & 0 & 0.0 & 5 & 0 & 0.0 & 62 & 0 & 0.0 & 5 & 0 & 0.0 & 62 & 0 & 0.0 \\
\hline$>3-15$ years & 9 & 5 & 55.6 & 224 & 14 & 6.3 & 9 & 3 & 33.3 & 224 & 28 & 12.5 & 9 & 4 & 44.4 & 224 & 46 & 20.5 \\
\hline Juveniles & 2 & 0 & 0.0 & 52 & 0 & 0.0 & 2 & 2 & 100.0 & 52 & 22 & 42.3 & 2 & 1 & 50.0 & 52 & 4 & 7.7 \\
\hline Young adults & 3 & 2 & 66.7 & 80 & 6 & 7.5 & 3 & 3 & 100.0 & 80 & 40 & 50.0 & 3 & 2 & 66.7 & 80 & 8 & 10.0 \\
\hline Middle adults & 7 & 7 & 100.0 & 186 & 16 & 8.6 & 7 & 6 & 85.7 & 186 & 72 & 38.7 & 7 & 5 & 71.4 & 186 & 28 & 15.1 \\
\hline Old adults & 7 & 5 & 71.4 & 123 & 24 & 19.5 & 7 & 3 & 42.9 & 123 & 21 & 17.1 & 7 & 3 & 42.9 & 123 & 18 & 14.6 \\
\hline Total & 33 & 19 & 57.6 & 727 & 60 & 8.3 & 33 & 17 & 51.5 & 727 & 183 & 25.2 & 33 & 15 & 45.5 & 727 & 104 & 14.3 \\
\hline \multirow[t]{2}{*}{ Cluster B - earthen } & \multicolumn{6}{|c|}{ Caries } & \multicolumn{6}{|c|}{ Calculus } & \multicolumn{6}{|c|}{ LEH } \\
\hline & $\mathrm{Ni}$ & $n$ & $\%$ & $\mathrm{Nt}$ & $n$ & $\%$ & $\mathrm{Ni}$ & $n$ & $\%$ & $\mathrm{Nt}$ & $n$ & $\%$ & $\mathrm{Ni}$ & $n$ & $\%$ & $\mathrm{Nt}$ & $n$ & $\%$ \\
\hline Juveniles & 9 & 3 & 33.3 & 257 & 6 & 2.3 & 9 & 9 & 100.0 & 257 & 117 & 45.5 & 9 & 1 & 11.1 & 257 & 7 & 2.7 \\
\hline Young adults & 4 & 2 & 50.0 & 112 & 6 & 5.4 & 4 & 4 & 100.0 & 112 & 37 & 33.0 & 4 & 0 & 0.00 & 112 & 0 & 0.0 \\
\hline Middle adults & 5 & 4 & 80.0 & 115 & 18 & 15.7 & 5 & 4 & 80.0 & 115 & 36 & 31.3 & 5 & 2 & 40.0 & 115 & 21 & 18.3 \\
\hline Total & 18 & 9 & 50.0 & 484 & 30 & 6.2 & 18 & 17 & 94.4 & 484 & 190 & 39.3 & 18 & 3 & 16.7 & 484 & 28 & 5.8 \\
\hline
\end{tabular}

$N i$ total number of individuals, $N t$ total number of teeth observed, $n$ total number of individuals/teeth affected

While limited pathological information has been published elsewhere (see Price et al. 2017), the work on complete skeletal pathologies from the site is currently ongoing by one of the authors (YB) and will be presented fully in future publications. However, as part of this study, a sample of skeletons from the site was analysed for dental lesions. Sample size consisted of 1540 teeth, originating from 67 of the 99 osteologically analysed skeletons with teeth (Table 2). Caries, calculus and linear enamel hypoplasia (LEH) were recorded according to Buikstra and Ubelaker (1994). The distribution of lesions in cluster B (coffins versus earthen burials, sex and age groups) was examined. Comparisons between cluster A and B were omitted, due to the small sample size in cluster A, and suspected representation issues, except for comparisons that could be made between sub-adults $(0$ 15 years) in the two clusters.

\section{Methods}

Bone samples from a total of 67 skeletons from Salberget cemetery were analysed for stable carbon and nitrogen isotopes. Mostly ribs were selected for analysis (Table 3), avoiding pathological bones as these have shown to potentially affect isotopic data determined (see for instance Katzenberg and Lovell 1999; Olsen et al. 2014). The bone samples belong to children $(n=22)$, juveniles ( $n=12$, including nine from earthen burials) and adults ( $n=33$, including nine from earthen burials). This sample comprises approximately half of the total number of children that were osteologically analysed $(n=43)$, almost all of the total number of juveniles $(n=15)$ and approximately two thirds of all adults identified $(n=44)$. The sample selected for isotope analysis is made up of over half of the coffin burials and more than two thirds of the earthen burials (55.7 and $81.8 \%$ respectively).

Bone collagen was extracted using standard protocols based on Longin (1971), with modifications recommended by Brown et al. (1988). For operational reasons, samples were prepared at both the Archaeological Research Laboratory in Stockholm (AFL, 2009 and 2013) and at the Tandem Laboratory in Uppsala (TL, 2010, see Table 3). The differences in methods are noted in brackets in the following text. At AFL, the sampled powder was first demineralised in a $0.25 \mathrm{M} \mathrm{HCl}$ solution over $\mathrm{c}$. $48 \mathrm{~h}$ in room temperature to make the inorganic part of the bone dissolve ( $\mathrm{TL}-0.8 \mathrm{M} \mathrm{HCl}$ solution for $30 \mathrm{~min}$ ). The first solution was then filtrated and rinsed three times with de-ionised water. A $0.01 \mathrm{M} \mathrm{HCl}$ solution was then added to the sample and then put into the oven at $58^{\circ} \mathrm{C}$ for approximately $16 \mathrm{~h}$ to gelatinize (TLwater was added, and the sample was heated in $90{ }^{\circ} \mathrm{C}$ under stirring for 6-8 h). The samples were then filtrated into ultrafilters and centrifuged in order to collect the collagen in its liquid form. Isotope ratios were determined using EA/CF-IRMS at the UC Davis Stable Isotope Facility, Department of Plant Sciences, Davis, California, USA, and at the Stable Isotope Laboratory at the Department for Geological Sciences at Stockholm University, Sweden (in the case of the samples prepared in 
Table 3 Stable carbon and nitrogen isotope data from human bone collagen from Salberget cemetery $(n=67)$, including $\delta^{13} \mathrm{C}, \delta^{15} \mathrm{~N}, \% \mathrm{C}, \% \mathrm{~N}$ and $\mathrm{C}: \mathrm{N}$, alongside details of burial type and location

\begin{tabular}{|c|c|c|c|c|c|c|c|c|c|c|c|}
\hline $\mathrm{Lab}$ & Sample & Bone & $\delta^{13} \mathrm{C}_{\text {coll. }}(\mathrm{V}-\mathrm{PDB}, \%$ ) & $\delta^{15} \mathrm{~N}_{\text {coll. }}(\mathrm{AIR}, \%$ $)$ & $\% \mathrm{C}$ & $\% \mathrm{~N}$ & $\mathrm{C}: \mathrm{N}$ & Age group & Sex & Coffin & Location \\
\hline AFL & 2531 & Fibula & -19.8 & 15.5 & 44.2 & 15.4 & 3.4 & Juvenile & $?$ & $\mathrm{X}$ & A \\
\hline AFL & 2549 & Femur & -20.2 & 15.5 & 44.0 & 15.5 & 3.3 & Old adult & ㅇ? & $\mathrm{X}$ & A \\
\hline AFL & 2581 & Fibula & -20.3 & 16.1 & 44.6 & 16.1 & 3.2 & Older child & $?$ & $\mathrm{X}$ & A \\
\hline AFL & 2599 & Ulna & -20.2 & 15.6 & 43.4 & 15.6 & 3.2 & Younger child & $?$ & $\mathrm{X}$ & $\mathrm{A}$ \\
\hline AFL & 2616 & Fibula & -20.1 & 15.8 & 43.9 & 15.8 & 3.2 & Older child & $?$ & $\mathrm{X}$ & A \\
\hline AFL & 2633 & Fibula & -20.6 & 11.8 & 35.4 & 11.8 & 3.5 & Middle adult & q & $\mathrm{X}$ & A \\
\hline AFL & 2646 & Talus & -20.1 & 15.8 & 42.8 & 15.1 & 3.3 & Old adult & ふ?? & $\mathrm{X}$ & A \\
\hline AFL & 2659 & Ulna & -20.2 & 15.1 & 42.8 & 15.1 & 3.3 & Older child & $?$ & $\mathrm{X}$ & A \\
\hline AFL & 2804 & Fibula & -19.7 & 15.5 & 43.2 & 15.5 & 3.3 & Young adult & ㅇ? & $\mathrm{X}$ & A \\
\hline AFL & 3117 & $\mathrm{Rib}$ & -20.0 & 12.6 & 37.7 & 13.9 & 3.2 & Juvenile & $?$ & $\mathrm{X}$ & A \\
\hline AFL & 3362 & Rib & -19.7 & 13.3 & 44.6 & 16.6 & 3.1 & Older child & $?$ & $\mathrm{X}$ & A \\
\hline AFL & 3377 & Rib & -20.0 & 12.7 & 44.1 & 16.3 & 3.2 & Older child & $?$ & $\mathrm{X}$ & A \\
\hline AFL & 3777 & Rib & -20.2 & 12.9 & 43.4 & 15.8 & 3.2 & Young adult & ㅇ? & $\mathrm{X}$ & $\mathrm{A}$ \\
\hline AFL & 3790 & Fibula & -20.3 & 15.0 & 42.7 & 15.0 & 3.3 & Middle adult & ¿’? & $\mathrm{X}$ & A \\
\hline AFL & 3818 & Fibula & -20.0 & 15.8 & 44.2 & 15.8 & 3.3 & Older child & $?$ & $\mathrm{X}$ & A \\
\hline AFL & 4031 & Rib & -19.4 & 13.6 & 45.4 & 16.8 & 3.1 & Middle adult & ¿’? & $\mathrm{X}$ & A \\
\hline AFL & 4086 & Fibula & -19.7 & 15.6 & 43.7 & 15.6 & 3.3 & Old adult & $\hat{\sigma}$ & $\mathrm{X}$ & A \\
\hline AFL & 4105 & Tibia & -20.6 & 12.2 & 37.7 & 12.2 & 3.6 & Old adult & §? & $\mathrm{X}$ & A \\
\hline AFL & 4135 & Rib/scapula & -20.6 & 15.5 & 43.4 & 15.5 & 3.3 & Middle adult & q? & $\mathrm{X}$ & A \\
\hline AFL & 5023 & Fibula & -20.6 & 16.1 & 44.4 & 16.1 & 3.2 & Younger child & $?$ & $\mathrm{X}$ & A \\
\hline AFL & 5498 & Fibula & -20.7 & 15.3 & 43.1 & 15.3 & 3.3 & Old adult & $0^{2}$ & $\mathrm{X}$ & A \\
\hline $\mathrm{TL}$ & 5860 & Rib & -20.3 & 12.3 & 39.5 & 14.0 & 3.3 & Middle adult & q & $\mathrm{X}$ & B1 \\
\hline AFL & 5914 & Rib & -19.6 & 11.9 & 42.5 & 15.6 & 3.2 & Middle adult & त? & & $\mathrm{C} 1$ \\
\hline AFL & 5926 & Pars petrosa & -19.4 & 12.5 & 41.0 & 14.9 & 3.2 & Middle adult & त? & & $\mathrm{C} 1$ \\
\hline AFL & 6037 & Rib & -19.5 & 12.0 & 43.8 & 16.2 & 3.2 & Middle adult & $\hat{\sigma}$ & $\mathrm{X}$ & B1 \\
\hline $\mathrm{TL}$ & 6072 & Rib/ulna & -20.0 & 13.5 & 35.1 & 12.6 & 3.3 & Old adult & q & $\mathrm{X}$ & B1 \\
\hline TL & 6110 & Cranium & -20.7 & 13.0 & 35.1 & 12.3 & 3.3 & Old adult & q & $\mathrm{X}$ & B1 \\
\hline AFL & 6123 & Rib & -19.6 & 11.7 & 43.6 & 16.1 & 3.2 & Young adult & $\hat{0}$ & $\mathrm{X}$ & B1 \\
\hline $\mathrm{TL}$ & 6135 & Tibia & -20.4 & 12.9 & 25.0 & 8.7 & 3.4 & Juvenile & $?$ & $\mathrm{X}$ & B1 \\
\hline AFL & 6160 & Rib & -19.4 & 12.2 & 42.1 & 15.4 & 3.2 & Young adult & $\hat{\sigma}$ & & $\mathrm{C} 1$ \\
\hline AFL & 6279 & Scaphoideum & -20.3 & 12.7 & 43.8 & 15.9 & 3.2 & Old adult & ㅇ? & $\mathrm{X}$ & $\mathrm{C} 2$ \\
\hline AFL & 6315 & Rib & -19.7 & 11.5 & 39.8 & 14.6 & 3.2 & Juvenile & ¿? & & $\mathrm{C} 1$ \\
\hline TL & 6684 & Rib/cranium & -19.9 & 15.5 & 38.7 & 13.9 & 3.3 & Younger child & $?$ & $\mathrm{X}$ & B1 \\
\hline TL & 6709 & Scapula & -20.1 & 13.1 & 33.3 & 11.9 & 3.3 & Old adult & q & $\mathrm{X}$ & B1 \\
\hline TL & 6842 & Rib/fibula & -20.4 & 12.7 & 32.6 & 11.8 & 3.2 & Juvenile & ¿?? & & $\mathrm{C} 1$ \\
\hline TL & 6846 & Rib & -19.9 & 12.6 & 34.1 & 12.4 & 3.2 & Middle adult & $\hat{\sigma}$ & & $\mathrm{C} 1$ \\
\hline $\mathrm{TL}$ & 6850 & Rib & -20.0 & 12.5 & 36.6 & 13.3 & 3.2 & Juvenile & $\hat{\sigma}$ & & $\mathrm{C} 1$ \\
\hline AFL & 6854 & Rib & -19.8 & 12.3 & 44.1 & 16.3 & 3.2 & Juvenile & ¿’? & & $\mathrm{C} 1$ \\
\hline TL & 6858 & Rib & -19.1 & 12.8 & 35.2 & 12.9 & 3.2 & Young adult & $\hat{\sigma}$ & & $\mathrm{C} 1$ \\
\hline AFL & 6862 & Rib & -20.1 & 12.3 & 43.9 & 15.6 & 3.3 & Juvenile & त? & & $\mathrm{C} 1$ \\
\hline AFL & 6866 & Rib & -20.0 & 12.0 & 43.9 & 16.2 & 3.2 & Juvenile & त? & & $\mathrm{C} 1$ \\
\hline TL & 6870 & Rib & -20.1 & 12.0 & 23.5 & 8.3 & 3.3 & Juvenile & त? & & $\mathrm{C} 1$ \\
\hline $\mathrm{TL}$ & 6912 & Humerus & -20.5 & 13.4 & 33.4 & 11.6 & 3.4 & Younger child & $?$ & $\mathrm{X}$ & B1 \\
\hline $\mathrm{TL}$ & 6943 & Rib & -19.3 & 12.4 & 37.6 & 13.6 & 3.2 & Middle adult & $\hat{0}$ & & $\mathrm{C} 1$ \\
\hline AFL & 6951 & Rib & -20.1 & 13.1 & 43.0 & 15.8 & 3.2 & Older child & $?$ & $\mathrm{X}$ & B1 \\
\hline $\mathrm{TL}$ & 6990 & Rib/clavicle & -18.6 & 14.1 & 33.9 & 12.2 & 3.2 & Middle adult & $\hat{o}$ & & $\mathrm{C} 1$ \\
\hline AFL & 6994 & Rib & -20.2 & 10.8 & 36.0 & 13.5 & 3.1 & Young adult & $\hat{0}$ & & $\mathrm{C} 1$ \\
\hline $\mathrm{TL}$ & 7031 & Fibula & -20.7 & 12.5 & 28.7 & 10.2 & 3.3 & Older child & $?$ & $\mathrm{X}$ & B1 \\
\hline
\end{tabular}


Table 3 (continued)

\begin{tabular}{|c|c|c|c|c|c|c|c|c|c|c|c|}
\hline Lab & Sample & Bone & $\delta^{13} \mathrm{C}_{\text {coll. }}(\mathrm{V}-\mathrm{PDB}, \%$ ) & $\delta^{15} \mathrm{~N}_{\text {coll. }}(\mathrm{AIR}, \%$ o $)$ & $\% \mathrm{C}$ & $\% \mathrm{~N}$ & $\mathrm{C}: \mathrm{N}$ & Age group & Sex & Coffin & Location \\
\hline TL & 7035 & Ulna & -20.9 & 13.5 & 32,0 & 11.0 & 3.4 & Younger child & $?$ & $\mathrm{X}$ & B1 \\
\hline AFL & 7134 & Rib & -19.0 & 13.2 & 43.6 & 15.9 & 3.2 & Juvenile & o? & & B2 \\
\hline TL & 7139 & Parietal/fibula & -20.3 & 11.5 & 34.1 & 12.5 & 3.2 & Juvenile & $\hat{\sigma}$ & & B2 \\
\hline TL & 7143 & Rib & -19.5 & 13.2 & 31.8 & 11.6 & 3.2 & Young adult & o? & & B2 \\
\hline AFL & 7464 & Tibia & -20.6 & 13.1 & 43.1 & 15.2 & 3.3 & Old adult & $?$ & $\mathrm{X}$ & $\mathrm{D}$ \\
\hline AFL & 7519 & Rib & -20.4 & 11.6 & 44.2 & 16.5 & 3.1 & Older child & $?$ & $\mathrm{X}$ & $\mathrm{D}$ \\
\hline AFL & 7555 & Rib & -20.1 & 12.4 & 43.0 & 15.8 & 3.2 & Older child & $?$ & $\mathrm{X}$ & $\mathrm{D}$ \\
\hline AFL & 7665 & Rib & -20.3 & 13.0 & 44.7 & 16.3 & 3.2 & Younger child & $?$ & $\mathrm{X}$ & $\mathrm{D}$ \\
\hline AFL & 7669 & Parietal & -20.1 & 15.1 & 44.0 & 15.9 & 3.1 & Younger child & $?$ & $\mathrm{X}$ & $\mathrm{D}$ \\
\hline AFL & 7770 & Rib & -20.6 & 12.1 & 43.2 & 15.4 & 3.3 & Older child & $?$ & $\mathrm{X}$ & $\mathrm{D}$ \\
\hline AFL & 7825 & Metacarpal V & -20.0 & 13.1 & 44.0 & 16.4 & 3.1 & Old adult & ㅇ? & $\mathrm{X}$ & $\mathrm{D}$ \\
\hline AFL & 7866 & Rib & -19.9 & 13.3 & 41.6 & 16.0 & 3.0 & Young adult & o & $\mathrm{X}$ & $\mathrm{D}$ \\
\hline AFL & 7874 & Rib & -20.3 & 14.0 & 45.3 & 16.8 & 3.1 & Younger child & $?$ & $\mathrm{X}$ & $\mathrm{D}$ \\
\hline AFL & 7879 & Rib & -20.2 & 12.0 & 44.3 & 16.1 & 3.2 & Older child & $?$ & $X$ & D \\
\hline AFL & 7980 & Rib & -20.0 & 12.7 & 44.4 & 16.1 & 3.2 & Middle adult & क? & $\mathrm{X}$ & D \\
\hline AFL & 7984 & Rib & -20.1 & 13.6 & 43.4 & 15.3 & 3.3 & Younger child & $?$ & $\mathrm{X}$ & $\mathrm{D}$ \\
\hline AFL & 7988 & Rib & -20.6 & 11.4 & 43.4 & 15.9 & 3.2 & Older child & $?$ & $\mathrm{X}$ & $\mathrm{D}$ \\
\hline AFL & 8107 & Rib & -19.8 & 13.4 & 44.3 & 16.4 & 3.2 & Middle adult & $\hat{\sigma}$ & $\mathrm{X}$ & $\mathrm{D}$ \\
\hline AFL & 8238 & Rib & -19.8 & 12.8 & 40.5 & 14.9 & 3.2 & Middle adult & $\widehat{0}$ & $X$ & $\mathrm{D}$ \\
\hline
\end{tabular}

TL Tandem Laboratory, Ångström Laboratory, Uppsala University, Sweden, AFL Archaeological Research Laboratory, Stockholm University, Sweden

Stockholm) and at the Tandem Laboratory in Uppsala. The combined analytical precision across all analyses was $\pm 0.2 \%$ or better $(1 \sigma)$ for both $\delta^{13} \mathrm{C}$ and $\delta^{15} \mathrm{~N}$. Although there were some discrepancies between the specific details of preparation techniques used (e.g. acid concentration, duration) and analyses (e.g. instrumentation, in-house standards, etc.) in the different laboratories, as indicated by recent modest and larger-scale studies (e.g. Jørkov 2007; Pestle 2010; Pestle et al. 2014; Sealy et al. 2014; Rand et al. 2015), these are unlikely to have influenced the quality of isotope data determined and are therefore considered interpretively insignificant.

As faunal isotope values are necessitated to provide 'baselines' for the interpretation of human dietary isotope data (Britton et al. 2008; Eriksson 2013), six faunal samples, collected at excavations of the Salberget cemetery and the mining village, were also selected for collagen extraction and isotope analysis. Unfortunately, only collagen extracted from two of these specimens (cattle and pig, see Table 4) passed published quality
Fig. 4 Carbon and nitrogen isotope ratiosin coffin and earthen burials, Salberget cemetery $(n=67)$

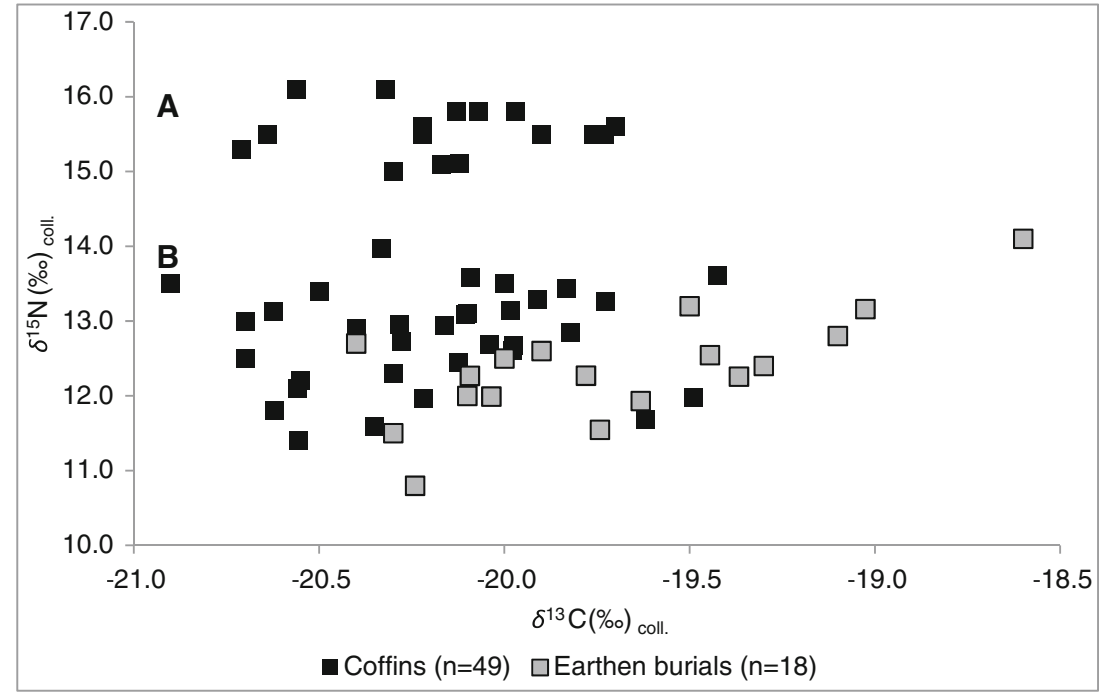


Table 4 Stable carbon and nitrogen isotope data from fauna from Salberget, including $\delta^{13} \mathrm{C}, \delta^{15} \mathrm{~N}, \% \mathrm{C}, \% \mathrm{~N}$ and $\mathrm{C}: \mathrm{N}$ data $(n=2)$

\begin{tabular}{|c|c|c|c|c|c|c|c|c|c|}
\hline Lab & Sample & Species & Bone & $\begin{array}{l}\delta^{13} \mathrm{C}_{\text {coll. }} \\
(\mathrm{V}-\mathrm{PDB}, \% o)\end{array}$ & $\begin{array}{l}\delta^{15} \mathrm{~N}_{\text {coll. }} \\
(\mathrm{AIR}, \% \text { ) }\end{array}$ & $\% \mathrm{C}$ & $\% \mathrm{~N}$ & $\mathrm{C}: \mathrm{N}$ & Age \\
\hline TL & F70 & Pig & Ulna SIN & -22.6 & 7.8 & 31.0 & 10.8 & 3.3 & Adult \\
\hline TL & F75 & Cattle & $\mathrm{C} 2+3 \mathrm{DX}$ & -22.3 & 5.1 & 36.1 & 12.7 & 3.3 & Adult \\
\hline
\end{tabular}

$T L$ Tandem Laboratory, Ångström Laboratory, Uppsala University, Sweden

control criteria (after Ambrose 1990; van Klinken 1999). Therefore, in order to enhance the understanding of local environmental background values, herbivore and omnivore isotope data from previously published Swedish sites of similar age are incorporated into this study including Viking age Birka (Linderholm et al. 2008) and medieval Visby, Sigtuna, Skänninge and Västerås (Lindkvist 2007; Kjellström et al. 2009; Arcini et al. 2014; Fjellström and Eriksson 2014). These studies also included isotope data for brackish and freshwater fish, like perch and pike, and Baltic and North Sea cod and herring were supplemented from Swedish, Polish and British medieval sites (Müldner and Richards 2005, 2007b; Lindkvist 2007; Barrett et al. 2008).

\section{Results}

All human bone collagen data generated in this study can be found in Table 3. Elemental $(\% \mathrm{C}, \% \mathrm{~N})$ data are consistent values expected for modern collagen (van Klinken 1999), aside from two samples (sample nos. 6135 and 6870) which have slightly low $\% \mathrm{C}$ and $\% \mathrm{~N}$. However, $\mathrm{C}: \mathrm{N}$ ratios for these individuals meet quality indicators (3.4 and 3.3, respectively) and are therefore considered acceptable. Similarly, a single sample had a C:N ratio exceeding 3.5 (sample no. 4105), although $\% \mathrm{C}$ and $\% \mathrm{~N}$ data are acceptable and the $\mathrm{C}: \mathrm{N}$ value (3.6) falls within the range proposed by Ambrose (1990). For the Salberget population as a whole $(n=67), \delta^{13} \mathrm{C}$ values range from -20.9 to $-18.6 \%$, with a mean and standard deviation of $-20.1 \pm 0.4 \%, 1 \sigma$. The range of $\delta^{15} \mathrm{~N}$ values was larger and spans from 10.8 to $16.1 \%$, with a mean and standard deviation of $13.3 \pm 1.4 \% o, 1 \sigma$. As seen in Fig. 4 , the carbon and nitrogen isotope ratios from Salberget cemetery are divided into two clearly separated groups (clusters A and B). This difference is significant at $p \leq 0.05$, using the MannWhitney $U$ test $\left(\delta^{15} \mathrm{~N}\right.$ values: $U$ value $1207.5, Z$ score -2.213 , $p$ value 0.027 ). A further difference can be seen between coffin (clusters $\mathrm{A}$ and $\mathrm{B}$ ) and earthen burials (cluster $\mathrm{B}) ; \delta^{13} \mathrm{C}$ values among the earthen burials are slightly enriched in comparison with both groups of coffin burials (Table 5). However, the difference in both $\delta^{13} \mathrm{C}$ and $\delta^{15} \mathrm{~N}$ values between the two groups ( $n=45$, children omitted from calculations) is not significant at $p \leq 0.05$ (Mann-Whitney $U$ test, $\delta^{13} \mathrm{C}$ values: $U$ value $941.5, Z$ score $-0.247, p$ value $0.803 ; \delta^{15} \mathrm{~N}$ values: $U$ value $829.5, Z$ score $1.117, p$ value 0.242 ).

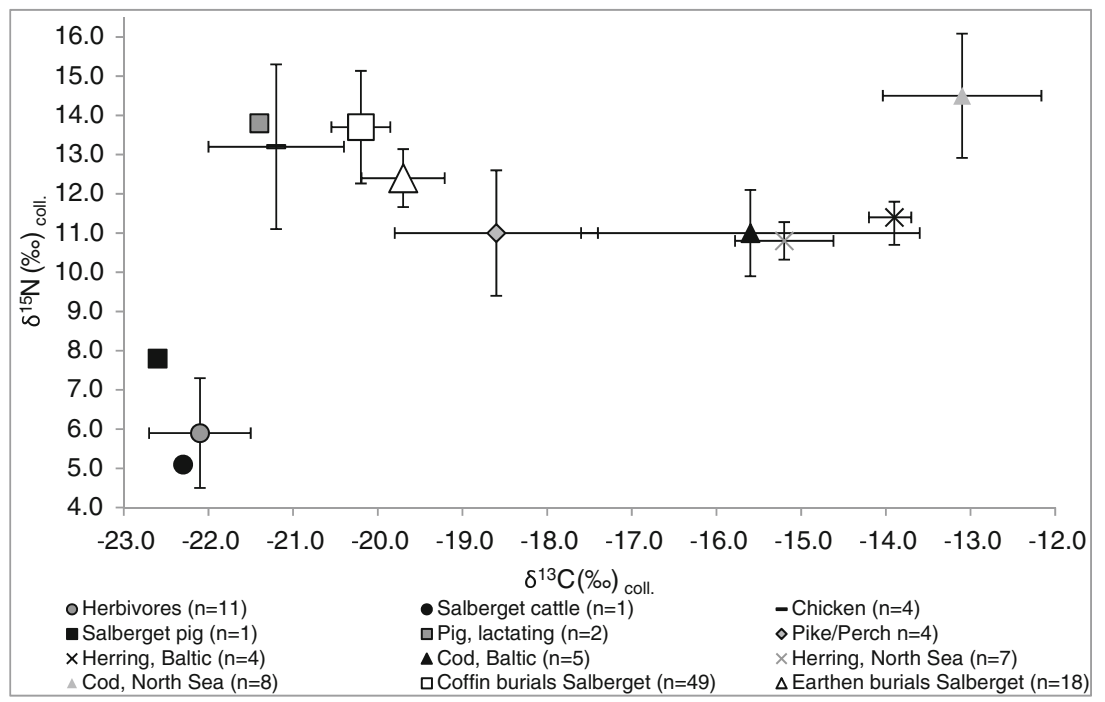

Fig. 5 Salberget stable carbon and nitrogen isotope mean values plotted with faunal data from Salberget mining village, and other proximal Swedish studies (faunal data taken from Müldner and Richards 2005, 2007b; Lindkvist 2007; Barrett et al. 2008; Linderholm et al. 2008;
Kjellström et al. 2009; Arcini et al. 2014; Fjellström and Eriksson 2014). The carbon values for Baltic herring $\left(\delta^{13} \mathrm{C}\right.$ mean $=-13.9 \pm 0.2 \%$, $1 \sigma$ ) are derived from the medieval town Skänninge in Östergötland, with an inland location c. $240 \mathrm{~km} \mathrm{SSW} \mathrm{of} \mathrm{Salberget} \mathrm{(Arcini} \mathrm{et} \mathrm{al.} \mathrm{2014)}$ 

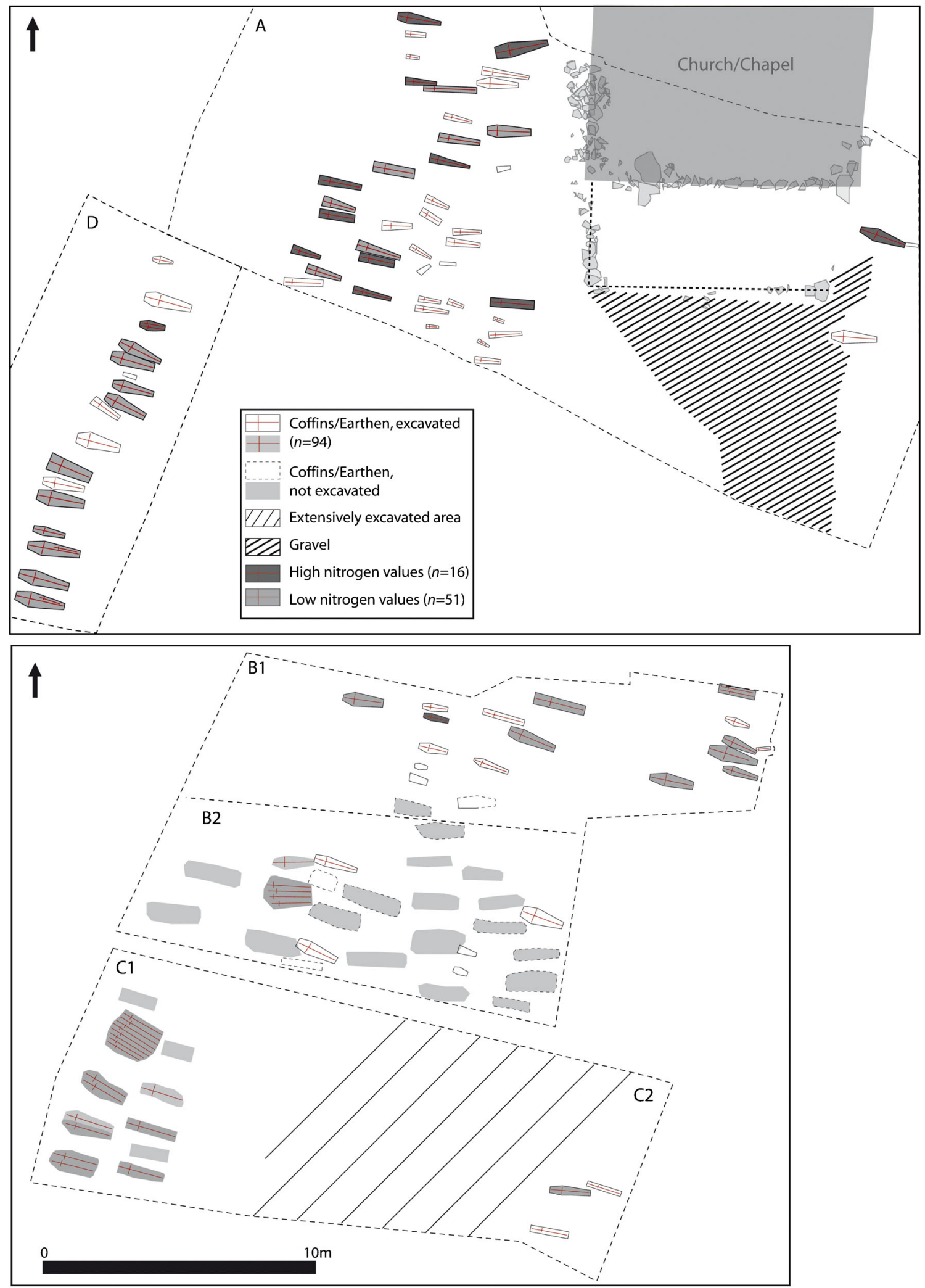

Fig. 6 Spatial distribution of skeletons sampled for $\delta^{13} \mathrm{C}$ and $\delta^{15} \mathrm{~N}$ analysis $(n=67)$. a Detail of excavated burials in trenches A and D. b Detail of excavated burials in trenches $\mathrm{B}$ and $\mathrm{C}$

Almost 90\% of the coffin burials in cluster A were found in trench A close to the church ( $n=14$ of 16 analysed samples), and almost $80 \%$ of the coffin burials in cluster B in trenches B1-2, C2 and D ( $n=26$ of 33 analysed samples). All of the 
Fig. 7 Distribution of livestock in AD 1620: 111 households in Salberget (left) and 122 peasant households in Sala parish (right) (Tax registers including livestock and arable land 1620-24)

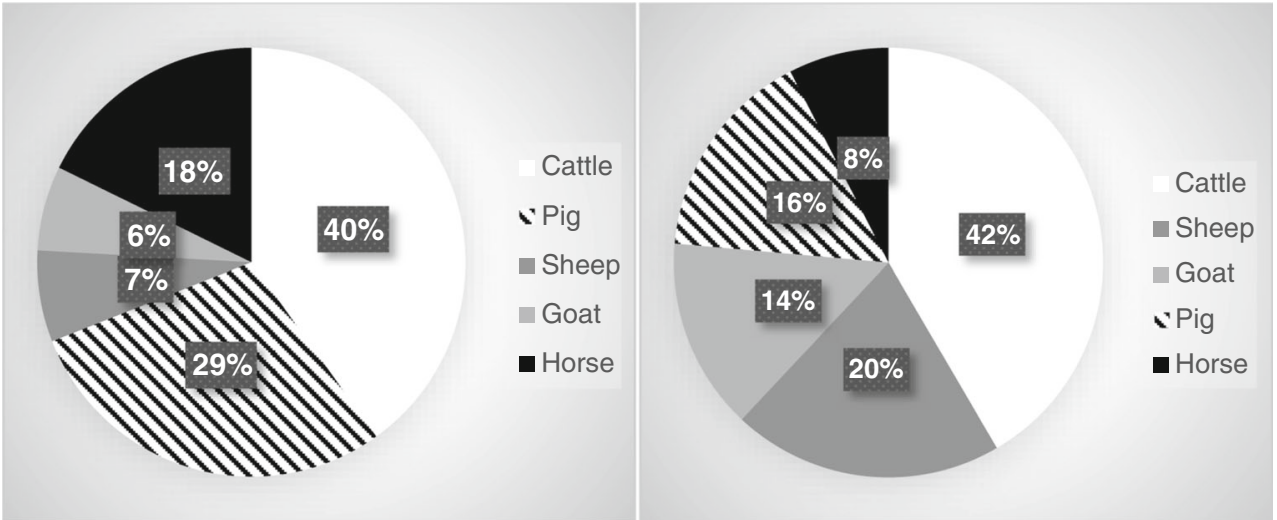

earthen burials in cluster B are found in trenches B2 and C1 (see Fig. 3).

In general, the protein in the diet of the mining population was dominated by terrestrial resources, albeit with some marine input and/or possible freshwater protein input. The mean $\delta^{15} \mathrm{~N}$ values $(13.3 \pm 1.4 \%$ o, $1 \sigma$, and $13.7 \pm 1.0 \%$, $1 \sigma$, respectively) are elevated relative to local fauna $(+8.2$ and $+5.5 \%$ or cattle and pig, respectively, Fig. 5). Usually, the trophic level enrichment for each step in the food chain is assumed to be between 3 and 5\%o, which means that the nitrogen mean value for the Salberget population is slightly high compared to anticipated local herbivore and even omnivore values. These higher nitrogen values imply a contribution of higher trophic level animal protein of some kind to the diet (for example, freshwater fish or other omnivorous species, such as poultry). Alternatively, this could also perhaps be due to the influence of soil improvement methods on the isotope ratios of crops and grasslands, for example, through manuring. Experiments by Bogaard et al. (2007) demonstrated an increase in $\delta^{15} \mathrm{~N}$ in manured winter wheat by as much as $3 \%$. However, this is not likely as the cattle reference from the mining cemetery do not show an elevated nitrogen isotope value $\left(\delta^{15} \mathrm{~N}=5.1 \%\right.$ ), which is similar to values determined from 10 herbivore bone samples collected from other medieval sites in the counties of Västmanland, Uppland and Östergötland (mean $=6.0 \pm 1.4 \%$, $1 \sigma$, see Linderholm et al. 2008; Kjellström et al. 2009; Arcini et al. 2014; Fjellström and Eriksson 2014, and Fig. 5), and it also correlates well with British herbivores from contemporary sites with nitrogen values normally ranging between c. 5 and 6\%o (Müldner and Richards 2007b).

\section{Dietary variation in the Salberget mining community: integrating isotopes, dental lesions, zooarchaeology and local historical sources}

In the following, dietary patterns in identities connected to age, sex, gender and status/occupation will be discussed. For the sex and age distribution in Salberget cemetery, see Table 1.
As mentioned before, two different groups were identified in the Salberget cemetery through stratigraphy and osteoarchaeology: earthen burials with juveniles and younger males in one part of the cemetery and coffin burials containing males, females, juveniles and children in others. The $\delta^{13} \mathrm{C}$ and $\delta^{15} \mathrm{~N}$ mean values separate the mining population into three different groups (see Fig. 4), one comprising the earthen buried males and the other two the coffin burials.

The coffin burials in cluster A are found in trench A close to the church/chapel (Fig. 6, see also Fig. 3) and clearly distinguished from the rest of the burials, displaying enriched nitrogen values in combination with depleted carbon values. The variation in the diet of those buried in cluster A seems to be rather small compared to the diet of the two other groups, where in particular the nitrogen values display a greater range. The large difference between herbivore and human $\delta^{15} \mathrm{~N}$ values at Salberget combined with terrestrial carbon isotope values indicates that, at least for cluster $\mathrm{A}$, protein in the diet likely contained higher trophic level omnivorous protein (pork - including from young animals - chicken, eggs, etc.) and/or freshwater fish. This interpretation can be supported by the quantification of species found among the animal refuse in the mining village, where pigs (piglets) and even poultry (eggs) were found in rather high numbers, although cattle dominate the faunal food waste (Carlsson 1987: 92-102). The combination of a diet containing a large proportion of animal protein and a burial location close to the church, both markers of high status (see for example Hagberg 1937; Daniell 1997; Hadley 2001), may be explained by their being of higher social status within the mining society.

In comparison to the demographically mixed population buried in coffins in clusters $\mathrm{A}$ and $\mathrm{B}$, the earthen buried males in cluster B have slightly less negative carbon isotope values, combined with elevated $\delta^{15} \mathrm{~N}$ values, which may suggest a marine component in their diet, similar to trends determined in some other Northern European medieval coastal communities (see for instance Müldner and Richards 2007a, b; Müldner et al. 2009; Yoder 2012; Curtis-Summers et al. 2014 (period 4); Quintelier et al. 2014). In this sense, and in dietary terms, the 
Fig. 8 The proportion of meat (black) and fish (white) served at Väsby estate in 1570 (calculations based on lispound) (Account books for Sala and Väsby demesne 1570: 3A)

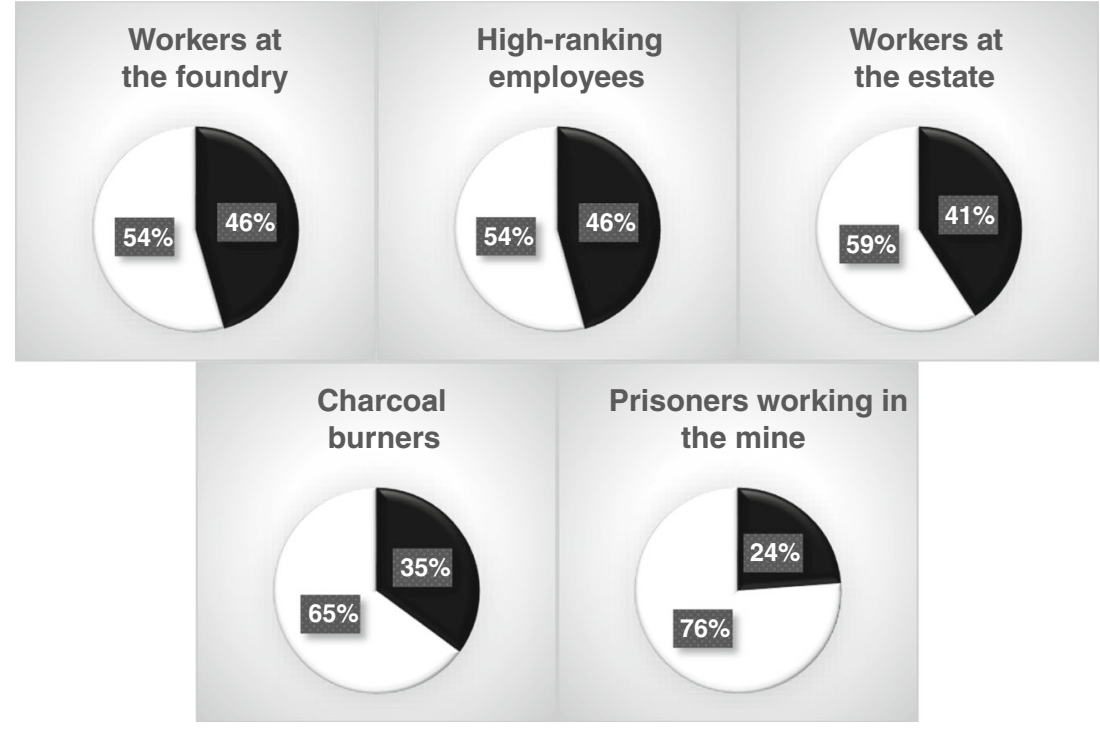

mining community seems to be a much more heterogeneous and socially stratified society than previously anticipated; this can be seen in the wide range of $\delta^{15} \mathrm{~N}$ values between 10.8 and $16.1 \%$ o (difference $5.3 \%$ ), for example.

The animal bone waste from the archaeological excavations of Salberget mining community and the vast contemporary archival material from Salberget provide a rare opportunity for both corroborating and (possibly) contradicting the interpretation of diet as inferred from the dietary isotopes. The importance of terrestrial domesticate protein in the diet is mirrored by the animal bone waste found in excavations of the mining village in the 1950s and $1980 \mathrm{~s}$ (Nordahl 1958; Bergold and Öhnegård 1987) and also by livestock registers from the sixteenth and early seventeenth century. The presence of other protein sources including fish and plant protein (such as legumes) is also indicated by food distribution lists and account books, as well as from tax registers listing declared goods sold at the local markets.

The animal bone waste from the mining village gives a picture of consumption patterns (Bergold and Öhnegård 1987; Carlsson 1987). Compared to the livestock registers from 1620 (Fig. 7 left), the villagers seem to have consumed less beef/veal $(\approx 30 \%)$ than they raised, approximately the same amount of pork, and more mutton and/ or goat meat $(\approx 40 \%)$. The osteological analysis by Ronnie Carlsson shows that most of the culling of cattle took place after the age of three, indicating that they primarily were kept to provide the households with dairy products and not meat. This could explain the discrepancy in proportions of cattle in registers and in excavation records (40\% cattle and around 30\%, respectively). Furthermore, most of the cattle (around $78 \%$ ), calves omitted, was made up by cows, compared to $58 \%$ in the surrounding farm scape of Sala parish, which give further support for the interpreted importance of dairy products. Furthermore, the bone waste shows that sheep and/or goats and pigs were slaughtered preferably under the age of 1, suggesting that the meat in their diet primarily consisted of pork and lamb. Meat from goats and/or sheep was probably bought from surplus production of sheep and/or goats by local farmers, which comprised over a third of their total livestock (Fig. 7, right). Support for this interpretation can be found in account books from 1618, showing that the meat that most of the female sorters bought from Väsby estate was beef (from oxen) and mutton. From the oldest building phase in the village (in between 1450 and 1510), the bone debris also include relatively large amounts of bones from birds, chicken $(\mathrm{MNI}=15)$ and geese $(\mathrm{MNI}=4){ }^{2}$ Bone refuse from roe deer and mountain hare in smaller quantities, and fish (not determined to species), were also found (Carlsson 1987: 92-102).

According to the livestock records, the households in the village had on average five to six animals each, including one horse (poultry excluded) (Älvborgs lösen 1571; Tax registers including livestock and arable land 1620-24). In tax registers for the village in 1620 (111 households), $40 \%$ of the livestock was cattle, almost $30 \%$ pigs, almost $20 \%$ horses, and $13 \%$ sheep and goats, respectively (Fig. 7 left). In Sala parish (122 households, the households in the mining village excluded) in the same year, there was on average 18 to 19 animals per household; $42 \%$ of the livestock was made up of cattle (297 oxen, steers and bulls, 557 cows and 103 young cattle between 1 and 3 years of age), $16 \%$ of pigs, $34 \%$ of sheep and goats and only $8 \%$ of horses (Fig. 7 right) (Tax registers including livestock and arable land 1620-24). Note that calves, lambs, young goats, piglets, rams and poultry were not consistently recorded in the tax registers.

\footnotetext{
${ }^{2} \mathrm{MNI}=$ minimum number of individuals.
} 


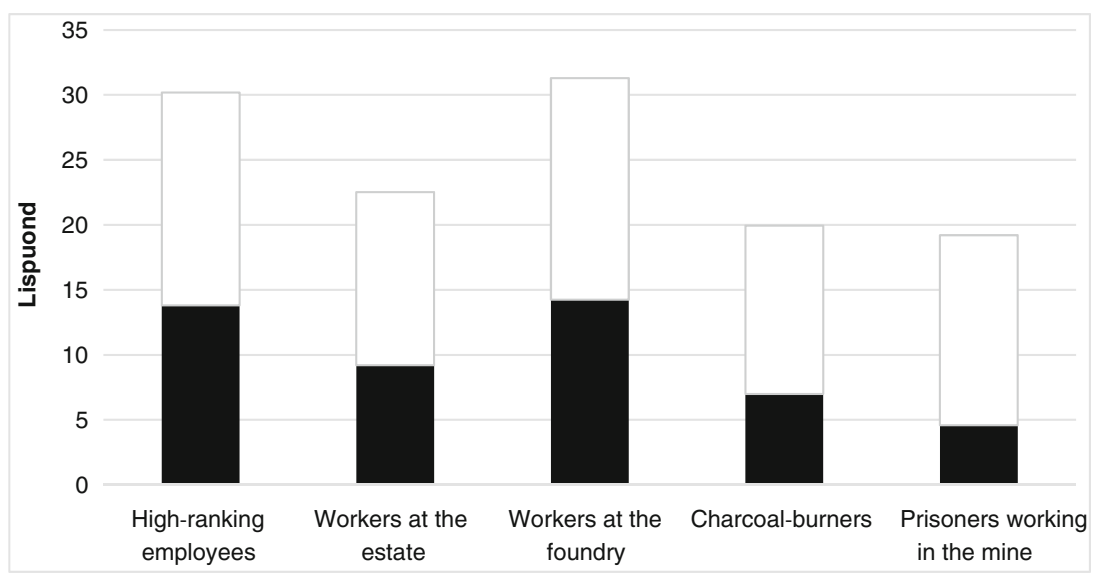

Fig. 9 The quantity of meat (black) and fish (white) in lispound ( 1 lispound $=6.65 \mathrm{~kg}$ for dried victuals and $6.06 \mathrm{~kg}$ for food in brine) served per person a year at each table (Account books for Sala and Väsby demesne 1570: 3A)

The food products consumed in the mining community seem mainly to originate from three sources: (1) the "miners" own allotments and livestock, (2) markets and (3) as served meals at Väsby estate. Not all the workers were served meals at the estate; for instance, the female sorters (sovrekvinnorna, sovre- = Eng. sort, sift, separate), separating lumps of silver ore from gauge, were not, and in general, nor the 'proper' miners. The food distribution lists from the estate display a hierarchy, foods being split into different menus based on status. In the following discussion, 'tables' for five different social groups are compared, high-ranking employees, workers at the foundry, workers at the estate, charcoal-burners and prisoners, although there are some additional tables, for instance the fishermen's table, that were excluded in this study due to methodological issues.

In January 1570, 48 to 53 people, including five women, were served weekly meals at the high-ranking employees table ${ }^{3}$, more than 60 people, whereof three women, at the foundry-workers' table, ${ }^{4}$ and 53 to 57 people ( 13 women) at the table of the workers of the estate. ${ }^{5}$ Beside these three relatively large groups, around 20 prisoners and six charcoal-burners had their own table regimes/menus (Account books for Sala and Väsby demesne 1570: 3A). Figure 8 presents the proportions of meat and fish served at different tables over the year (1570) at Väsby estate.

\footnotetext{
$\overline{3}$ Among them various kinds of bailiffs and managers (gårdsfogden, bergsfogden, underfogdar), clerks (kammarskrivaren, underskrivare) and other officials, servants to the bailiff of the estate, the King's servants, guards, the matron (fataburshustrun), the matron's maid (fataburspigan), mjölkdejan (a high-ranking female worker responsible for the entire farm production of animal products, and the breeding of animals), the bakeress and the housemistress (stugukvinnan).

${ }^{4}$ The supervisor of the foundry (hyttefogden), clerks (hytteskrivare), foundry workers (hytteknektar), a chef, a brewer, a carpenter, a blacksmith, refiners (drivare), smelters, dalkarlar, one dairy maid, one cattle maid, one housemistress (stugukvinna), etc. A few of them dined only 1, 2 or 3 weeks or were visiting temporarily.

${ }^{5}$ The Crown's blacksmith, 15 ore stooges (malmdrängar), 20 farm hands, eight matrons (fataburskvinnor), one bakeress, two cattle maids, two pig maids, one Danish and three Swedish prisoners.
}

The pie charts display a sliding scale between the different groups; the higher up the social ladder, the more meat was served (see discussion in Woolgar et al. 2006: 100 regarding diet in medieval England). The ratio of fish versus meat among highranking employees and workers at the foundry was almost 50/50, among the prisoners $75 / 25$.

The same pattern is seen in the amounts of meat and fish served (Fig. 9); the high-ranking employees and the workers at the foundry received significantly greater quantities of both fish and meat, approximately 30-35\% more than the prisoners and charcoal-burners. The same kind of differences is repeated in the food distribution registers for the year AD 1608 (Account books for Sala and Väsby demesne 1608). Furthermore, if we look into the details of the menus served at the different tables, there is a greater variety of foodstuffs at higher class tables. Above all, the differences in quantity and variation define different group diets, and a similar pattern is seen all over Europe during the medieval and post-medieval period (see for example Sykes 2006: 65). For example, in AD 1570 , there were at least 14 different types of fish served at the table of the high-ranking employees, including perch, dried eel and salted cod (and herring of course). Sea fish (mostly salted) outnumbered freshwater fish, but there were also minor contributions of fresh and dried freshwater fish, for example the fish caught by the permanent staff of fishermen at Väsby estate. Fresh fish was only served at the finer tables. At the prisoners' table, herring was the main fish consumed (and in larger quantities compared to meat). The dominance of sea fish over freshwater fish in the prisoners' diet is illustrated in Fig. 10 (Account books for Sala and Väsby demesne 1570: $3 \mathrm{~A})$. The marine signal seen among the earthen burials correlates well with the prisoners' diet. According to the records from AD 1544 and AD 1545, meat from poultry, i.e. geese and chicken, was only served at the 'finer' tables, i.e. the tables of the high-ranking employees' and the workers of the foundry, as well as meat from hare and mutton/lamb. In the same years, 


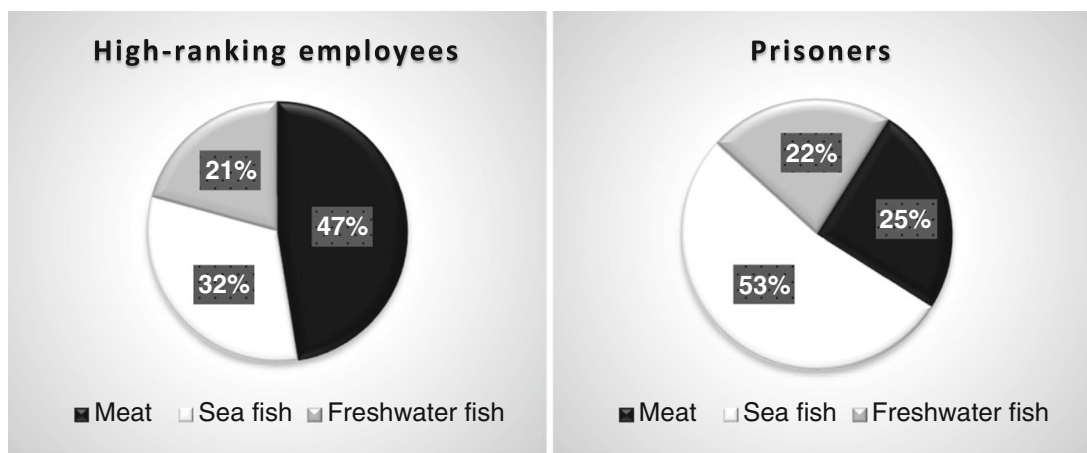

Fig. 10 Distribution of freshwater and sea fish at the high-ranking employees' and the prisoners' tables. Old measurement units converted to kilogrammes/person (Account books for Sala and Väsby demesne 1570: 3A)

beef and pork were served at every table (Account books for Sala and Väsby demesne 1544: 4, 1545: 11).

The various kinds of fish and meat in the diet were complemented by other animal and plant products, such as butter, milk, bread, beer and vegetables. Butter was served at every table but in varying amounts. Fresh milk, chickens and eggs were exclusively served at the high-ranking employees' table. At the two 'finer' tables, cheese was served but not in any larger quantities and just on few occasions throughout the year (see also discussion in Albarella 2006; Dyer 2006; Sykes 2006; Woolgar 2006). The gardens of the estate produced parsley root, parsnips, winter radish, carrots, onions, horseradish and hops, but only the last product can be traced in the menus from 1570. Although the account books where extremely detailed for other kinds of food, only the consumption of larger vegetables, such as cabbage and turnips, were recorded. In season, cabbage (around 9 months/ year) and turnips (5 to 6 months/year) were served at most of the tables (Account books for Sala and Väsby demesne 1570: 3A, 1594: 7).

In summary, the dietary isotopes present diverse diets for the skeletons buried in coffin and earthen burials, respectively. A marine contribution to the diet was found among the earthen males buried in the southern part of the cemetery, with a mainly terrestrial diet among the demographically diverse population buried in coffins albeit with differing mean nitrogen values between coffin cluster $\mathrm{A}$ and $\mathrm{B}\left(\delta^{15} \mathrm{~N}=15.6 \pm 0.3 \%\right.$, $1 \sigma$, and $\delta^{15} \mathrm{~N}=12.8 \pm 0.7 \%$ o, $1 \sigma$, respectively), splitting them into two groups. When considered alongside the documentary sources, correlations are found between earthen buried males and prisoners working in the mine being served all their meals by the estate, a diet containing approximately three quarters of sea fish (i.e. mainly herring) relative to meat and incorporating proteins from plant sources, which corresponds well with the marine diet indicated by the carbon isotope values compared to the remainder of the population. The prisoners'/earthen buried males diet resembles that of contemporary coastal populations from Scotland, Belgium and Orkney(see e.g. Polet and Katzenberg 2003; Richards et al. 2006; Curtis-Summers et al. 2014) more than the diet found among the Salberget population buried in the coffins and also in comparison with other Swedish populations from Linköping and Sigtuna (Kjellström et al. 2009; Arcini et al. 2014). The observed differences in terrestrial and marine protein in the diet in earthen and coffin burials, respectively, might, if not explained by social position in society, be connected to their being of different origin; Polish, Danish and Russian prisoners being mentioned in the written sources (see Bäckström and Price 2016). The correlation with other groups mentioned in the records, i.e. high-ranking employees, workers at the foundry, workers at the estate and charcoal-burners may be regarded as less certain, due to the lack of knowledge of their entire diet. Furthermore, the proper miners are not targeted, since they, in general, were not fed by the estate. However, these different menus may well be discussed and provide clues as to the diets of the populations from upper (cluster A) and lower social strata (cluster B) in the mining village. For instance, foodstuffs exclusively served at the high-ranking employees' table, such as poultry, eggs and eel compared to other groups, offer an explanation to the high nitrogen values among people buried close to the chapel/church in cluster A.

In addition to highlighting differences in the working males' diets, the isotope data provide insights into the diets of females, children, adolescents (15-20 years of age) and older adults (i.e. in this case +50 years) at the site, further illuminating societal differences. These groups, including those buried in coffins, seem to have included more terrestrial plant and animal than marine protein, compared to the earthen buried males and juveniles, and younger and middle adults buried in coffins (Fig. 11). In the study of dental pathologies, calculus is more commonly associated with individuals whose diets included more marine protein. The risk of getting calculus in the marine group is 2.2 times higher than in the terrestrial group. ${ }^{6}$ However, the association between a (presumably) high protein diet, rich in marine fish, and calculus may be casual rather than causal, and the influence of differential oral hygiene practices between the different groups cannot be excluded. The lowest $\delta^{15} \mathrm{~N}$ values can be found in children between 3 and 15 years of age and in adolescents and

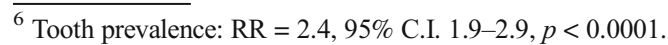


Fig. 11 Carbon and nitrogen isotope ratios in age groups $(n=51)$, Salberget cemetery. Cluster A omitted (YA young adult, 20-35 years, $M A$ middle adult, 35-50 years, $O A$ old adult, +50 years)

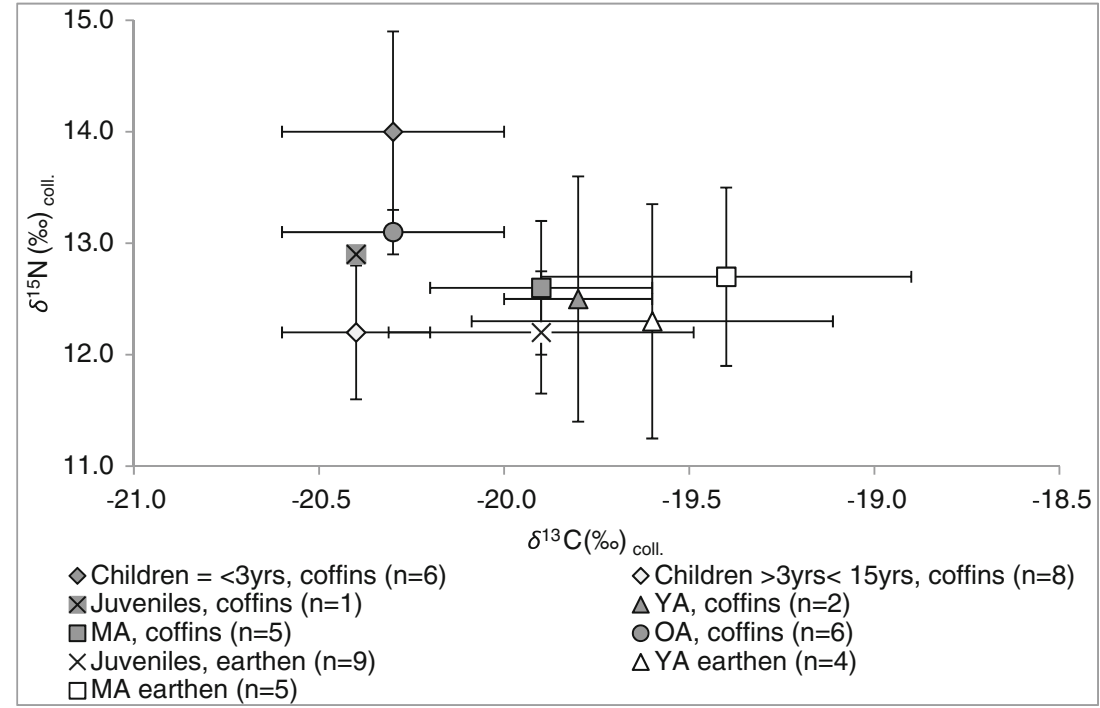

young adults (i.e. 20-35 years of age) in earthen burials, the highest in adults older than 50 years of age and children younger than 3 years of age. The elevated mean nitrogen value of children below the age of 3 years can in part be explained by breastfeeding (Richards et al. 2002). Note that cluster A (see Fig. 5), with mean nitrogen isotope ratios for the different age groups even more elevated than these age groups, is omitted from Fig. 11 due to small sample size.

A greater proportion of marine protein in the diet is indicated among the reproductively viable and able-bodied part of the population, from around 15 years of age up to 50 years of age. These differences in terrestrial versus marine input in the diet could be controlled by availability or by cultural customs/habits or by economic status. In this case, we might be able to connect this difference to the feeding at Väsby estate, since only working people was offered meals (as part of their salary). Some members of families, such as younger children, older people and females (to some extent), for example the female sorters mentioned earlier, were excluded. In the written documents, the people served at the different tables are registered but not their age, although we know that some minors under the age of 15 were listed on payrolls as well as some females (Ingvarsson et al. 2017). In accordance with this, females should have lower $\delta^{13} \mathrm{C}$ values consistent with a lower proportion of marine foods in their diet and that is also the case, although differences between males and females are slight (see Fig. 12).

Figure 12 shows that the diet of females in the low-status group (cluster B) to a greater extant was based on terrestrial plants and animals compared to males, or, in other words, the female diet was less marine, especially compared to the males
Fig. 12 Carbon and nitrogen isotope ratios in females $(n=11)$ and males $(n=21)$, Salberget cemetery

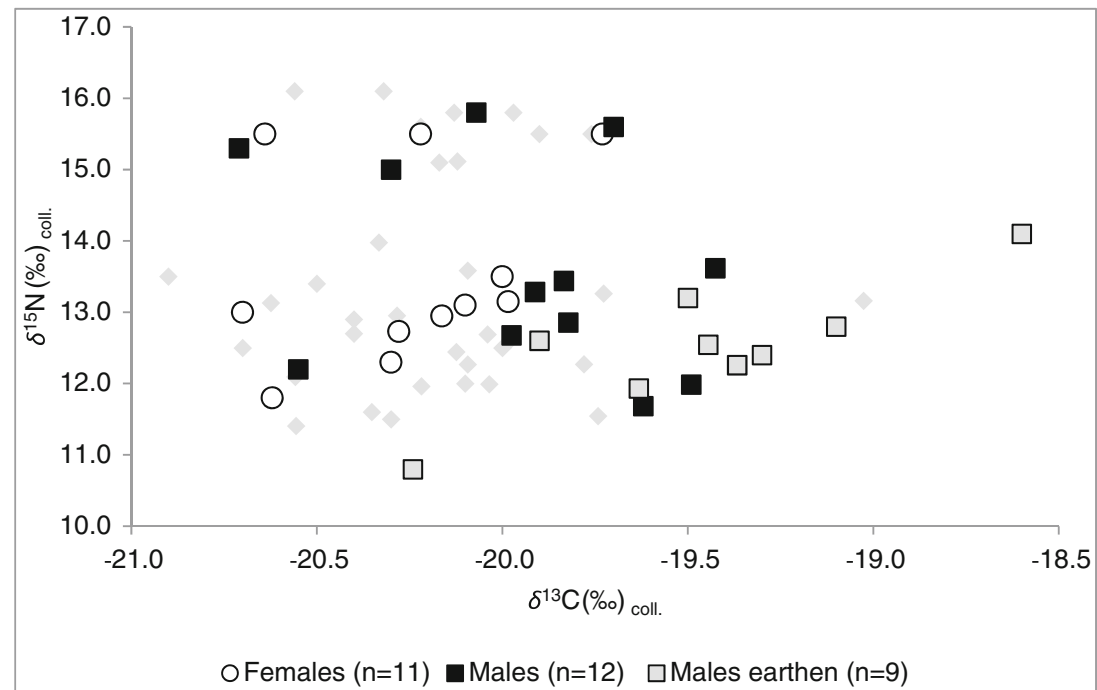


Table 5 Descriptive statistics (means and standard deviations) of carbon and nitrogen isotope data in clusters $\mathrm{A}$ and $\mathrm{B}$

\begin{tabular}{|c|c|c|c|c|c|}
\hline \multirow[b]{2}{*}{ Sample populations } & \multirow[b]{2}{*}{$N$} & \multicolumn{2}{|l|}{$\delta^{13} \mathrm{C}$} & \multicolumn{2}{|l|}{$\delta^{15} \mathrm{~N}$} \\
\hline & & Mean & $\operatorname{Std}(1 \sigma)$ & Mean & $\operatorname{Std}(1 \sigma)$ \\
\hline Coffins cluster A & 16 & -20.2 & 0.3 & 15.6 & 0.3 \\
\hline Coffins cluster B & 33 & -20.2 & 0.4 & 12.8 & 0.7 \\
\hline Earthen burials & 18 & -19.7 & 0.5 & 12.5 & 0.7 \\
\hline
\end{tabular}

buried in earthen burials. This pattern, of males consuming more marine resources than females, corresponds well with studies of late and post-medieval populations in Italy, Orkney, England, Denmark and Sweden (Müldner and Richards 2005, 2007a; Kjellström et al. 2009; Reitsema and Vercellotti 2012; Quintelier et al. 2014; Jørkov and Gröcke 2016). Moreover, the diet of females in cluster B was more homogenous than the diet of males. This distinction might be explained by a less varied diet by females eating at home compared to the menus served (mostly to males) at the estate. Furthermore, there are gender-related differences in diet between females and males in the 'low-status group' (coffins in cluster B) but notably not in the 'high-status group' (coffins in cluster A), showing that the dietary differences between sexes were governed by status. The prevalence of calculus is also higher among males than females and above all in males buried in coffins. ${ }^{7}$ Linear enamel hypoplasia is, in general, more frequent among females than among males, especially in comparison with the earthen males, indicating a more stressful environment (for example childhood fevers and dietary deficiencies) during childhood. ${ }^{8}$ This difference is also significant when comparing earthen and coffin burials in cluster B. ${ }^{9}$ Notably, the childhood of the earthen buried males and juveniles seems to have been the least stressful in comparison with the rest of the population. There are no major differences in caries rates between male and females.

\section{Conclusions}

In this paper, we applied documentary and bioarchaeological sources (i.e. isotope analysis of human and animal remains, dental pathologies and zooarchaeology) to investigate dietary patterns in a late medieval/early modern pre-industrial mining community in Salberget, Sweden. This approach provides new information about social structure, social identities and group diets and also demonstrates the benefits of using various

\footnotetext{
$\overline{7}$ Tooth prevalence calculus: cluster $\mathrm{A}+\mathrm{B}$ females $(n=11)$, cluster $\mathrm{A}+\mathrm{B}$ males $(n=21), \mathrm{RR}=1.7,95 \%$ C.I. $1.3-2.3, p=0.0001$; cluster $\mathrm{B}$ coffin males $(n=8)$, cluster B earthen males $(n=9), \mathrm{RR}=1.4,95 \%$ C.I. $1.1-1.7, p=0.02$.

${ }^{8}$ Tooth prevalence LEH: cluster A + B females $(n=11)$, cluster A + B males $(n=21), \mathrm{RR}=2.1,95 \%$ C.I. $1.4-3.0, p=0.0001$.

9 Tooth prevalence LEH: cluster B coffins $(n=33)$, cluster B earthen $(n=18)$, $\mathrm{RR}=2.5,95 \%$ C.I. $1.7-3.7, p<0.0001$.
}

source materials because they complement each other and enable a more complex interpretation.

The mining community, composed of different categories/groups of people, has proven to be more diverse and socially differentiated than expected, similar to the complexity of an urban community. The cemetery excavations and the subsequent skeletal analysis revealed two distinct groups: one buried in coffins, which in demographic composition resembles families or households, and the other with adolescents and males buried in earthen burials in a separate part of the churchyard. When adding the results of the isotope analyses, the people buried in coffins were split in two groups. The diet in both coffin groups was dominated by terrestrial protein (corresponding with Salbergets inland location), but the 'families' buried close to the church displayed high trophic level protein in their diet, and were interpreted as 'high-status'. In comparison to the coffin burials, the diet of the earthen buried males and juveniles exhibited more marine protein in their diet, similar to other coastal European populations. Connections were made between the earthen buried males and prisoners working in the mine catered by the Crown's estate - the dietary isotopes correlating with the documentary sources, as more marine fish were fed to the prisoners as part of their rations compared to the other menus served. Other aspects of the male earthen burials and their life-histories support this notion, with the 'prisoners' being largely non-local, items in the burial implying captivity (the iron-collar) and, for some, a violent death (as evidenced by un-healed fractures) (Price et al. 2017). A correlation was also found between the menus served at the two finer tables at Väsby estate and the isotopic composition of the 'highstatus' group in Salberget.

In addition, we determined dietary differences between females and males in the mining community with females consuming lower amounts of marine protein and exhibiting decreased dietary heterogeneity. Notably this was not the case in the 'high-status' group. Age-related differences were also observed: the diet of people over 50 years of age included more high trophic level protein from terrestrial/freshwater sources than the diets of other age groups, and the diet of the reproductively viable and able-bodied part of the population included a larger marine component, and in particular the earthen males, than the rest of the population. Furthermore, the diet among males, containing more marine protein, coincides with a higher prevalence of calculus.

Acknowledgements We would like to thank the Archaeological Research Laboratory, Stockholm University, Sweden and the Tandem Laboratory (Ångström Laboratory), Uppsala University, Sweden, for undertaking the analyses of stable nitrogen and carbon isotopes in both 
human and animal collagen samples. Also, thanks to Elin Ahlin Sundman for providing the $\delta^{13} \mathrm{C}$ and $\delta^{15} \mathrm{~N}$ values for animal references from Västerås.

This research (Bäckström's $\mathrm{PhD}$ employment at Lund University, Sweden) was supported by the Berit Wallenberg Foundation (BWS 2010.0176) and Jakob and Johan Söderberg's foundation. The 'Sala project' (excavations and analyses) has been funded by Riksens Clenodium, Jernkontoret, Birgit and Gad Rausing's Foundation, SAU's Research Foundation, the Royal Physiographic Society of Lund, Berit Wallenbergs Foundation, Åke Wibergs Foundation, Lars Hiertas Memory, Helge Ax:son Johnson's Foundation and The Royal Swedish Academy of Sciences.

Open Access This article is distributed under the terms of the Creative Commons Attribution 4.0 International License (http:// creativecommons.org/licenses/by/4.0/), which permits unrestricted use, distribution, and reproduction in any medium, provided you give appropriate credit to the original author(s) and the source, provide a link to the Creative Commons license, and indicate if changes were made.

\section{References}

Account books for Sala and Väsby demesne. In: Kammararkivet (KA), Landskapshandlingar, Västmanlands handlingar, 1544-1545, 1569-71, 1570, 1594, 1608

Tax registers including livestock and arable land. In: Boskaps- m.fl. längder, Västmanland vol. 2:4, 3, 4, 1620-24

Privileges of Salberget 2/7 1530 and 18/7 1535. In: Riksregistraturet, vol. $3-5,1529-36$

Printed Royal decree from 1526 concerning the mining area and supplies. In: "Kongl. Stadgar, förordningar, privilegier och resolutioner, angående justitien och hushållningen wid Bergwerken och Bruken... uti Sweriges Rike..." 1736, Stockholm

Salbergets accounts. In: KA, Särskilda serier utom reviderade räkenskaper, nr 511, vol. 77, 1602-03

Salberget accounts. In: KA, Bergsbruk, Räkenskaper för Salberget, 85:2, 1618

Account books and payrolls 1602-03. In: Ämnessamlingar 757.05, Handlingar rörande bergverken Sala bergslag vol. 2

Tax records Sala community, In: KA, Älvborgs lösen 1571, Västmanlands län

Adamson MW (2004) Food in medieval times. Food through history. Greenwood Press, Westport, pp 1-256

Albarella U (2006) Pig husbandry and pork consumption in medieval England. In: Woolgar C, Serjeantson D, Waldron T (eds) Food in Medieval England: history and archaeology. Oxford University Press, Oxford, pp 72-87

Alexander MM, Gerrard CM, Gutiérrez A, Millard AR (2015) Diet, society, and economy in late medieval Spain: stable isotope evidence from Muslims and Christians from Gandía, Valencia. Am J Phys Anthropol 156:263-273

Ambrose SH (1990) Preparation and characterization of Bone tooth collagen for isotopic analysis. J Archaeol Sci 17(4):431-451

Ambrose SH (2000) Controlled diet and climate experiments on nitrogen isotope ratios of rats. In: Ambrose SH, Katzenberg MA (eds) Biogeochemical approaches to Palaeodietary analysis. Kluwer Academic/Plenum Press, New York, pp 243-259

Ambrose SH, Norr L (1993) Experimental evidence for the relationship of the carbon isotope ratios of whole diet and dietary protein to those of bone collagen and carbonate. In: Lambert JB, Grupe G (eds) Prehistoric human Bone: archaeology at the molecular level. Springer-Verlag, New York, pp 1-37

Arcini C, Ahlström T, Tagesson G (2014) Variations in Diet and Stature: Are They Linked? Bioarchaeology and Paleodietary Bayesian
Mixing Models from Linköping, Sweden. Int J Osteoarchaeol 24: 543-556

Bäckström Y, Ingvarsson-Sundström A (2010) Sala Gruvkyrkogård. Arbete och straff vid Sala silvergruva. Etapp 2. SAU rapport 10:185

Bäckström Y, Ingvarsson-Sundström A (2012) Sala Gruvkyrkogård. Innanför och utanför vid Sala silvergruva. Etapp 3. SAU rapport 14:1-77

Bäckström Y, Price D (2016) Social identity and mobility at a preindustrial mining complex, Sweden. J Archaeol Sci 66:154-168

Bäckström Y, Ingvarsson-Sundström A, Onsten-Molander A (2009) Sala Gruvkyrkogård. Liv och död vid Sala silvergruva. Etapp 1. SAU rapport 2:1-40

Barrett J, Johnstone C, Harland J, Van Neer W, Ervynck A, Makowiecki D, Heinrich D, Hufthammer AK, Bødker Enghoff I, Amundsen C, Schou Christiansen J, Jones AKG, Locker A, Hamilton-Dyer S, Jonsson L, Lougas L, Roberts C, Richards M (2008) Detecting the medieval cod trade: a new method and first results. J Archaeol Sci $35: 850-861$

Bell LS, Lee Thorp JA, Elkerton A (2009) The sinking of the Mary rose warship: a medieval mystery solved? J Archaeol Sci 36:166-173

Bergold H, Öhnegård V (1987) Sala gruvby. Ett industrisamhälle från 1500-talet. Sala Kommun, Sala, pp 1-141

Bocherens H, Drucker D (2003) Trophic level isotopic enrichment of carbon and nitrogen in bone collagen: case studies from recent and ancient terrestrial ecosystems. Int J Osteoarchaeol 13:46-53

Boethius B (1951) Gruvornas, hyttornas och hamrarnas folk: bergshanteringens arbetare från medeltiden till gustavianska tiden. Tidens Förlag, Stockholm

Bogaard A, Heaton THE, Poulton P, Merbach I (2007) The impact of manuring on nitrogen isotope ratios in cereals: archaeological implications for the reconstruction of diet and crop management practices. J Archaeol Sci 34:335-343

Britton K, Müldner G, Bell M (2008) Stable isotope evidence for salt-marsh grazing in the bronze age Severn estuary, UK: implications for palaeodietary analysis at coastal sites. J Archaeol Sci 35(8):2111-2118

Brown TA, Nelson DE, Vogel JS, Southon JR (1988) Improved collagen extraction by modified Longin method. Radiocarbon 30:171-177

Buikstra JE, Ubelaker DH (eds) (1994) Standards for Data Collection from Human Skeletal Remains. Arkansas Archaeological Survey Research Series No 44, Fayetteville, pp 1-206

Carlsson R (1987) VIII. Benfynden ur osteologisk synpunkt. In: Bergold H, Öhnegård V (eds) Sala gruvby. Ett industrisamhälle från 1500talet. Sala Kommun, Sala, pp 92-102

Ciaffi R, Lelli R, Müldner G, Stantcheva K, Fischetti AL, Ghini G, Craig OE, Milano F, Rickards O, Arcudi G, Martínez-Labarga C (2015) Palaeobiology of the medieval population of Albano (Rome, Italy): a combined morphological and biomolecular approach. Int $\mathrm{J}$ Osteoarchaeol 25(4):477-488

Curtis-Summers S, Montgomery J, Carver M (2014) Stable isotope evidence for dietary contrast between Pictish and medieval populations at Portmahomack, Scotland. Mediev Archaeol 58:21-43

Daniell C (1997) Death and burial in medieval England 1066-1550. Routledge, London, pp 1-264

DeNiro MJ, Epstein S (1978) Influence of diet on the distribution of carbon isotopes in animals. Geochim Cosmochim Acta 42:495-506

Deniro M, Epstein S (1981) Influence of diet on the distribution of nitrogen isotopes in animals. Geochim Cosmochim Acta 45:341-351

Dyer CC (2006) Seasonal patterns in food consumption in the later middle ages. In: Woolgar C, Serjeantson D, Waldron T (eds) Food in medieval England: history and archaeology. Oxford University Press, Oxford, pp 201-214

Eriksson G (2013) Stable isotope analysis of humans. In: Tarlow S, Nilsson Stutz L, Belfer-Cohen A (eds) The Oxford handbook of the archaeology of death and burial. Oxford University Press, Oxford, pp 123-146 
Eriksson L, Berg L (2003) Salberget : en sammanställning över de bergshistoriska lämningarna i Fläckebo, Kila, Kumla, Möklinta, Norrby, Sala, Tortuna och Tärna socknar samt Sala stad. Atlas över Sveriges bergslag. Jernkontorets bergshistoriska utskott. H, 1101-5284; 118. Stockholm: Jernkontoret, pp 1-265

Fjellström M (2011) Stable isotope analysis and ethical issues surrounding a human skeleton material from Rounala in Karesuando parish. Master thesis, Stockholm University: Archaeological Research Laboratory, pp 1-53

Fjellström M, Eriksson G (2014) Kol-, kväve- och svavelisotopanalyser av humant och animalt skelettmaterial från Dominikanerkonventet, Västerås stad, Västmanland. Arkeologiska forskningslaboratoriet, Auxilia Uppdragsrapport, Stockholm, p 244

Froehle AW, Kellner CM, Schoeninger MJ (2010) FOCUS: effect of diet and protein source on carbon stable isotope ratios in collagen: follow up to Warinner and Tuross (2009). J Archaeol Sci 37:2662-2670

Hadley DM (2001) Death in medieval England. Tempus, Stroud, pp 1192

Hagberg L (1937) När döden gästar: svenska folkseder och svensk folktro i samband med död och begravning. Wahlström \& Widstrand, Stockholm, pp 1-733

Hedges REM, Clement JG, Thomas CDL, O'Connell TC (2007) Collagen turnover in the adult femoral mid-shaft: modelled from anthropogenic radiocarbon tracer measurements. Am J Phys Anthropol 133:808-816

Hillson SW (1979) Diet and dental disease. World Archaeol 11:2 Food and Nutrition:147-162

Iacumin P, Galli E, Cavalli F, Cecere L (2014) C4-consumers in southern Europe: the case of friuli VG (NE-Italy) during early and central middle ages. Am J Phys Anthropol 154(4):561-574

Ingvarsson A, Mispelaere J, Bäckström Y (2017) Above and below the surface: environment, work, death and upbringing in sixteenth- to seventeenth-century Sweden. In: Crawford, S, Hadley DM, Shepherd G (eds) Oxford handbook of the archaeology of childhood. Oxford: Oxford University Press, Handbook Series

Institute of Urban History, Stockholm (2008) Västerås befolkningsutveckling 1570-1995. Folkmängd i Västerås 1570 1850, http://ortshistoria.se/stad/vasteras/befolkning\#1 (2008-0121)

Jansson A (1963) Ur Sala gruvbys historia. Sala: Sala guideklubb, pp 1121

Jiménez-Brobeil S, Laffranchi Z, Maroto R, Sánchez FL, Huertas AD (2016) How royals feasted in the court of Pedro I of castile: a contribution of stable isotope study to medieval history. J Archaeol Sci Rep 10:424-430

Johansen OS, Gulliksen S, Nydal R (1986) $\Delta^{13} \mathrm{C}$ and diet: analysis of Norwegian human skeletons. Radiocarbon 28(2A):754-761

Jørkov MLS (2007) Evaluating bone collagen extraction methods for stable isotope analysis in dietary studies. J Archaeol Sci 34(11): 1824-1829

Jørkov MLS, Gröcke DR (2016) Investigating adult diet during Industrialization in Copenhagen based on stable isotope analysis of bone collagen and hair keratin. Archaeol Anthropol Sci, 15 pages, doi:10.1007/s12520-016-0373-5

Joyce RA (2005) Archaeology of the body. Annu Rev Anthropol 34:139158

Katzenberg MA, Lovell NC (1999) Stable isotope variation in pathological Bone. Int J Osteoarchaeol 9:316-324

Kjellström A, Storå J, Possnert G, Linderholm A (2009) Dietary patterns and social structures in medieval Sigtuna, Sweden, as reflected in stable isotope values in human skeletal remains. J Archaeol Sci 36: 2689-2699

Knudson KJ, Stojanowski CM (2008) New directions in Bioarchaeology: recent contributions to the study of human social identities. J Archaeol Res 16:397-432. doi:10.1007/s10814-008-9024-4
Lamb AL, Melikian M, Ives R, Evans J (2012) Multi-isotope analysis of the population of the lost medieval village of Auldhame, east Lothian, Scotland. J Anal At Spectrom 27(5):765-777

Lewis ME (2013) Children of the golden minster: St. Oswald's priory and the impact of industrialisation on child health. J Anthropol 2013, Article ID 959472:11. doi:10.1155/2013/959472

Lidén K, Nelson ED (1994) Stable carbon isotopes as dietary indicator, in the Baltic area. Fornvännen 89(1):13-21

Lieverse AR (1999) Diet and the Aetiology of dental calculus. Int J Osteoarchaeol 9:219-232

Linderholm A, Hedenstierna Jonson C, Svensk O, Lidén K (2008) Diet and status in Birka: stable isotopes and grave goods compared. Antiguity 82:446-461

Lindkvist J (2007) Kyrkotillhörighet och diet i det medeltida Visby. Diet och social status utifrån isotopanalyser av gravlagda från tre Visbykyrkor. Uppsats Arkeologi, CD. Stockholms Universitet, Stockholms, pp 1-40

Longin R (1971) New method of collagen extraction for radiocarbon dating. Nature 230:241-242

Lukacs JR (2011) Oral health in past populations: context, concepts and controversies. In: Grauer AL (ed) A companion to paleopathology. Wiley Blackwell companions to anthropology. Wiley, Chichester, pp 553-581

Makarewicz CA, Sealy J (2015) Dietary reconstruction, mobility, and the analysis of ancient skeletal tissues: expanding the prospects of stable isotope research in archaeology. J Archaeol Sci 56:146-158

Malmstedt G (1994) Helgdagsreduktionen. Övergången från ett medeltida till ett modernt år i Sverige 1500-1800. Avhandlingar från Historiska institutionen i Göteborg 8:1-285

Mays SA (1997) Carbon stable isotope ratios in mediaeval and later human skeletons from Northern England. J Archaeol Sci 24:561567

Meurman R (2000) Silverberg i Järnbärarland. Bergshanteringens begynnelse i ljuset av Schmidt Testhammardateringar. Occasional papers in archaeology. Acta Universitatis Upsaliensis, Uppsala, pp $1-184$

Mispelaere J, Lindström J (2015) Vad fick 1600-talets arbetare i lön? Historisk tidskrift 135(3):432-463

Morell M (1986) Eli F. Heckscher, utspisningsstaterna och den svenska livsmedelskonsumtionen från 1500-talet till 1800-talet. Sammanfattning och komplettering av en lång debatt. Uppsala Papers in Economic History, Research Report no 11. Department of Economic History, Uppsala, pp 1-50

Morell M (1989) Studier i den svenska livsmedelkonsumtionens historia: hospitalhjonens livsmedelskonsumtion 1621-1872, Uppsala: Acta Universitatis Upsaliensis, pp 1-354

Morell M (2011) Changes in food consumption. In: Myrdal J, Morell M (eds) Agrarian history of Sweden: from 4000 BC to AD 2000. Nordic Academic Press, Lund, p 2

Müldner G (2005) Eboracum-Jorvik-York: a diachronic study of human diet in York by stable isotope analysis. Dissertation, University of Bradford, pp 1-230

Müldner G, Richards MP (2005) Fast or feast: reconstructing diet in later medieval England by stable isotope analysis. J Archaeol Sci 32:39-48

Müldner G, Richards MP (2007a) Diet and diversity at later medieval Fishergate: the isotopic evidence. Am J Phys Anthropol 134:162 174

Müldner G, Richards MP (2007b) Stable isotope evidence for 1500 years of human diet at the City of York, UK. Am J Phys Anthropol 133: 682-697

Müldner G, Montgomery J, Cook G, Ellam R, Gledhill A, Lowe C (2009) Isotopes and individuals: diet and mobility among the medieval bishops of Whithorn. Antiquity 83:1119-1133

Norberg P (1978) Sala Gruvas Historia under 1500- och 1600-talen. Sala: Sala kommun, pp 1-646 
Nordahl E (1958) Rapport rörande 1955-1957-års undersökning av Sala gruvby. Unpublished report, Dnr 2878/58

Novak M, Martinci O, Strinovic D, Slaus M (2012) Skeletal and dental indicators of health in the late mediaeval (12-15th century) population from Nin, southern Croatia. HOMO - Journal of Comparative Human Biology 63:435-450

Olsen KC, White CD, Longstaffe FJ, von Heyking K, McGlynn G, Grupe G, Rühli FJ (2014) Intraskeletal isotopic compositions (d13C, $\mathrm{d} 15 \mathrm{~N}$ ) of Bone collagen: Nonpathological and pathological variation. Am J Phys Anthropol 153:598-604

Olsen KC, White CD, Longstaffe FJ, Rühli FJ, Warinner C, Salazar-García DC (2016) Isotopic anthropology of rural German medieval diet: intra- and inter-population variability. Archaeol Anthropol Sci:1-13

Onsten-Molander A, Jonsson K (2005) Sala Gruvkyrkogård. Arkeologisk förundersökning. SAU Skrifter 6:1-28

Palubeckaité Z, Jankauskas R, Boldsen J (2002) Enamel hypoplasia in Danish and Lithuanian late medieval/early modern samples: a possible reflection of child morbidity and mortality patterns. Int $\mathrm{J}$ Osteoarchaeol 12:189-201

Pestle WJ (2010) Chemical, elemental, and isotopic effects of acid concentration and treatment duration on ancient bone collagen: an exploratory study. J Archaeol Sci 37(12):3124-3128

Pestle WJ, Crowley BE, Weirauch MT (2014) Quantifying interlaboratory variability in stable isotope analysis of ancient skeletal remains. PLoS One 9(7):e102844

Polet C, Katzenberg MA (2003) Reconstruction of the diet in a mediaeval monastic community from the coast of Belgium. J Archaeol Sci 30: 525-533

Price TP, Frei R, Bäckström Y, Frei KM, Ingvarsson-Sundström A (2017) Origins of inhabitants from the $16^{\text {th }}$ century Sala (Sweden) silver mine cemetery - a lead isotope perspective. J Archaeol Sci 80:1-13

Quintelier K, Ervynck A, Müldner G, Van Neer W, Richards MP, Fuller BT (2014) Isotopic Examination of Links Between Diet, Social Differentiation, and DISH at the Post-medieval Carmelite Friary of Aalst, Belgium. Am J Phys Anthropol 153:203-213

Rand A, Bower M, Munkittrick J, Harris A, Burchell M, Grimes V (2015) Comparison of three bone collagen extraction procedures: the effect of preservation on $\delta^{13} \mathrm{C}$ and $\delta^{15} \mathrm{~N}$ values. North Atlantic Archaeology 4:93-113

Reitsema LJ, Vercellotti G (2012) Stable isotope evidence for sex- and status-based variations in diet and life history at medieval Trino Vercellese, Italy. Am J Phys Anthropol 148:589-600

Reitsema LJ, Crews DE, Polcyn M (2010) Preliminary evidence for medieval Polish diet from carbon and nitrogen stable isotopes. J Archaeol Sci 37(7):1413-1423

Richards MP, Mays S, Fuller BT (2002) Stable carbon and nitrogen isotope values of Bone and teeth reflect weaning age at the medieval Wharram Percy site, Yorkshire, UK. Am J Phys Anthropol 119: 205-210

Richards MP, Fuller BT, Molleson TI (2006) Stable isotope palaeodietary study of humans and fauna from the multi-period (iron age, Viking and late medieval) site of Newark Bay, Orkney. J Archaeol Sci 33: $122-131$

Salazar-Garcia DC, Richards MP, Nehlich O, Henry AG (2014) Dental calculus is not equivalent to bone collagen for isotope analysis: a comparison between carbon and nitrogen stable isotope analysis of bulk dental calculus, bone and dentine collagen from same individuals from the medieval site of el Raval (Alicante, Spain). J Archaeol Sci 47:70-77

Salesse K, Dufour E, Castex D, Velemínsky P, Santos F, Kucharova H, Jun L, Brûzek J (2013) Life history of the individuals buried in the St. Benedict cemetery (Prague, 15 th -18 th centuries): insights from $14 \mathrm{C}$ dating and stable isotope $(\mathrm{d} 13 \mathrm{C}, \mathrm{d} 15 \mathrm{~N}, \mathrm{~d} 18 \mathrm{O})$ analysis. Am J Phys Anthropol 151:202-214

Schoeninger MJ, DeNiro MJ (1984) Nitrogen and carbon isotopic composition of bone collagen from marine and terrestrial animals. Geochim Cosmochim Acta 48:625-639

Schoeninger MJ, DeNiro MJ, Tauber H (1983) Stable nitrogen isotope ratios of bone collagen reflect marine and terrestrial components of prehistoric human diet. Science 220:1381-1383

Sealy J, Johnson M, Richards M, Nehlich O (2014) Comparison of two methods of extracting bone collagen for stable carbon and nitrogen isotope analysis: comparing whole bone demineralization with gelatinization and ultrafiltration. J Archaeol Sci 47:64-69

Sundin J, Tedebrand L-G (2008) Dödlighet och sjuklighet i svensk järnbruksmilhö 1750-1875. Scandia: Tidskrift för historisk forskning 47(1):67-108

Sykes N (2006) From Cu and Sceap to Beffe and Motton. In: Woolgar C, Serjeantson D, Waldron T (eds) Food in medieval England: history and archaeology. Oxford University Press, Oxford, pp 56-71

Utterström G (1978) Fattig och föräldralös i Stockholm på 1600- och 1700-talen. Umeå Studies in the Humanities, Umeå, pp 1-248

Van Klinken GJ (1999) Bone collagen quality indicators for palaeodietary and radiocarbon measurements. J Archaeol Sci 26(6):687-695

Wåghäll Nivre E, Larsson O (2001) Reformationstiden. Kultur och samhällsliv i Luthers Europa. Studentlitteratur, Lund, pp 1-164

Warinner C, Tuross N (2009) Alkaline cooking and stable isotope tissuediet spacing in swine: archaeological implications. J Archaeol Sci 36: 1690-1697

Whittaker DK, Molleson T, Nuttall T (1998) Calculus deposits and bone loss on the teeth of Romano-British and eighteenth-century Londoners. Arch Oral Biol 43:941-948

Wild EM, Arlamovsky KA, Golser R, Kutschera W, Priller A, Puchegger S, Rom W, Steier P, Vycudilik W (2000) 14C dating with the bomb peak: an application to forensic medicine. Nucl Instrum Methods Phys Res B 172:944-950

Woolgar C (2001) Fast and feast: conspicuous consumption and the diet of the nobility in the fifteenth century. In: Hicks M (ed) The fifteenth century 2. Revolution and consumption in late medieval England. The Boydell Press, Woodbridge, pp 7-26

Woolgar C (2006) Group diets in Late Medieval England. In: Woolgar C, Serjeantson D, Waldron T (eds) Food in Medieval England: history and archaeology. Oxford University Press, Oxford, pp 191-200

Woolgar C, Serjeantson D, Waldron T (eds) (2006) Food in Medieval England: history and archaeology. Oxford, Oxford University Press, pp $1-347$

Yoder C (2010) Diet in medieval Denmark: a regional and temporal comparison. J Archaeol Sci 37(9):2224-2236

Yoder C (2012) Let them eat cake? Status-based differences in diet in medieval Denmark. J Archaeol Sci 39:1183-1193

Zvelebil M, Weber AW (2013) Human bioarchaeology: group identity and individual life histories - introduction. J Anthropol Archaeol $32: 275-279$ 